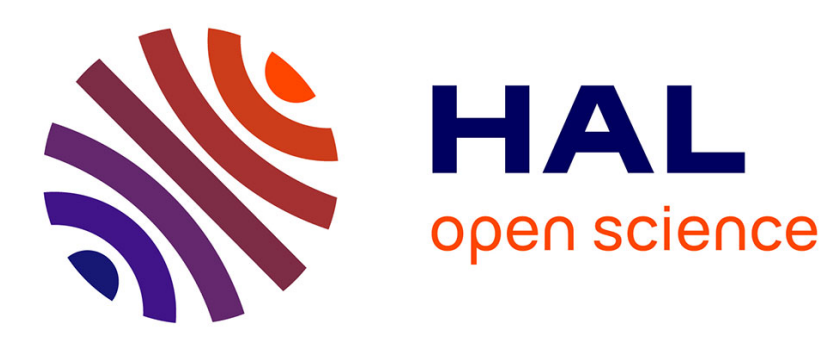

\title{
La géographie russe (1845-1917) à l'ombre et à la lumière de l'historiographie soviétique \\ Olivier Orain
}

\section{To cite this version:}

Olivier Orain. La géographie russe (1845-1917) à l'ombre et à la lumière de l'historiographie soviétique.

Espace Géographique, 1996, XXV (3), pp.217-232. halshs-00082222

\section{HAL Id: halshs-00082222 \\ https://shs.hal.science/halshs-00082222}

Submitted on 27 Jun 2006

HAL is a multi-disciplinary open access archive for the deposit and dissemination of scientific research documents, whether they are published or not. The documents may come from teaching and research institutions in France or abroad, or from public or private research centers.
L'archive ouverte pluridisciplinaire HAL, est destinée au dépôt et à la diffusion de documents scientifiques de niveau recherche, publiés ou non, émanant des établissements d'enseignement et de recherche français ou étrangers, des laboratoires publics ou privés. 


\title{
Olivier ORAIN
}

\section{LA GEOGRAPHIE RUSSE (1845-1917) À L'OMBRE ET À LA LUMIERE DE L'HISTORIOGRAPHIE SOVIÉTIQUE}

\author{
Notre Empire de Russie est une entité géographique, ce qui veut dire un \\ morceau d'une planète bien connue. Et l'Empire de Russie se compose de : \\ premièrement, la Grande Russie, la Petite-Russie, la Russie-Blanche et la \\ Russie ruthénienne; deuxièmement, des royaumes de Géorgie, de Pologne, \\ de Kazan et d'Astrakhan ; troisièmement, il comprend... Eh bien, et \\ caetera, et caetera.
}

Andreï Biélyi, Petersbourg, 1916.

La géographie russe prérévolutionnaire est quasiment inconnue en France, où elle n'a pas suscité le même intérêt qu'aux États-Unis ${ }^{1}$. Peut-être faut-il voir là le reflet d'une difficulté d'accès linguistique et, jusqu'à il y a peu, politique. Pourtant, le fonds russe est riche et a suscité localement une production historiographique abondante. Pendant soixante-quinze ans, les géographes soviétiques ont écrit un nombre important d'ouvrages sur le passé prérévolutionnaire de leur discipline. L'étude de cette production à notre connaissance sans équivalent constitue une introduction, sinon un préalable, à la découverte d'un corpus russe pré-soviétique tout à fait exceptionnel par sa variété et la vitalité scientifique dont il témoigne.

La profusion du discours historiographique confronte le chercheur étranger à la question de la finalité du retour aux origines et l'amène à établir des parallèles avec sa propre tradition de recherche. En France, l'historiographie disciplinaire a connu de multiples métamorphoses en l'espace d'un siècle. Jusqu'à la génération de Paul Vidal de la Blache, la discipline a connu une forte tradition de géographie historique, qui se faisait volontiers histoire de la géographie par le biais de la critique des sources. Avec les postvidaliens ${ }^{2}$, cette tradition s'est éclipsée au profit d'une référence diffuse aux précurseurs (et surtout au père fondateur) ayant pour objectif une légitimation de la discipline et une canonisation des pratiques de recherche. La contestation de la géographie dite classique dans les années soixante-dix a suscité une relecture critique des prédécesseurs et, de là, un "retour aux sources » qui a permis l'émergence d'une historiographie non plus anecdotique et légitimante, mais spécialisée et en quelque sorte professionnelle. Cette rénovation s'appuie sur des grilles de lecture qui doivent beaucoup à la sociologie des sciences et à l'historiographie des sciences humaines contemporaines. L'accent est mis sur l'inscription sociale de la discipline, la demande de savoir (ici géographique) dont est porteuse la société, les phénomènes d'institutionnalisation des sciences, tout ceci devant être confronté à l'évolution des contenus scientifiques.

Au regard de cette position de recherche, une double interrogation s'impose : quelles ont été les pratiques et les finalités de l'historiographie soviétique pendant trois quarts de siècle ? Et comment peut-on utiliser ce matériau en interaction avec les centres d'intérêt de l'historiographie occidentale actuelle pour comprendre l'histoire de la géographie russe au siècle dernier?

\footnotetext{
${ }^{1}$ Cf. notamment D. Hooson, «The Development Of Geography In Pre-soviet Russia », Ann. Amer. Geogr., 1968 ; M. Jeffrey \& J. Preston, All Possible Worlds, N.Y., 1972 ; W.B. Lincoln, Petr Petrovich Semënov-Tian Shanskï: The Life Of A Russian Geographer, Nextonville, Mass., 1980 ; M. Bassin, « Russian Geographers And The 'National Mission' In The Far East» in D. Hooson, Exsoviet identities and the return of geography, 1994, p. 112-133.

2 Par "postvidaliens », nous entendons les générations qui ont suivi celle de Vidal de la Blache et qui ont revendiqué l'héritage intellectuel de ce dernier.
} 


\section{Géographie impériale et historiographie soviétique}

En l'espace de soixante-dix ans, les géographes soviétiques ont publié une littérature historiographique étonnamment volumineuse ${ }^{3}$. Cette abondance de pages imprimées peut-elle nous faire signe?

\section{Ampleur du corpus}

Un recensement de deux mois nous a permis de relever plus de 90 références : plus de soixante livres et une trentaine d'articles ${ }^{4}$. Le caractère partiel de cet inventaire nous laisse à penser que l'on pourrait encore grossir la liste, sans parler de la production postsoviétique. À notre connaissance, rares sont les pays où l'on a accordé un tel intérêt à l'histoire disciplinaire. La profusion des publications n'est pourtant pas synonyme de diversité des contenus.

À lire ces ouvrages en série, on pense aux histoires littéraires du début du Xxe siècle : la restitution historique passe par une série de monographies individuelles (la vie et l'oeuvre...), généralement regroupées en chapitres, chaque chapitre essayant d'accréditer l'idée d'une école ou d'un courant par effet de juxtaposition. D'un ouvrage à l'autre, on retrouve à peu près les mêmes thèmes, les mêmes leitmotive, comme s'il y avait un canevas, une vulgate officiels à respecter. Parfois, cette disposition tourne au plagiat: la première biographie consacrée à Dimitri Nikolaiévitch Anoutchine (1843-1923), détenteur de la première chaire universitaire de géographie en Russie (1884), a été rédigée par son disciple Bogdanov en 19135. Cette notice a servi de banque de données - et d'idées (sans citation) - à la plupart de ses continuateurs. Dans les pages qu'il consacre à Anoutchine dans ses ouvrages de 1923 et 19496', l'éminent géographe (et historiographe) russe Liov Sémionovitch Berg (1876-1950) a poussé très loin l'art de l'imitatio Bogdanovi, reprenant les idées et l'organisation de l'original, voire plus. Au delà de cet exemple extrême, peut-être faut-il voir dans le caractère prolixe et répétitif de cette historiographie un résultat du provincialisme universitaire soviétique.

À partir des années 50 (rarement avant), les auteurs ont dû respecter un certain nombre de passages obligés de tout discours historisant en URSS, à commencer par la périodisation officielle de l'histoire pré-soviétique. Le découpage chronologique des chapitres suit obligatoirement celui de la terminologie lénino-stalinienne et chacun d'entre eux commence par un rappel général du contexte historique tel que Lénine l'avait théorisé. La Russie d'avant 1861, date de l'abolition du servage, est donc "féodale », dominée par un système "précapitaliste » et «absolutiste 》. Après 1861, l'Empire russe entre dans une phase de développement industriel et capitaliste, pour devenir finalement «impérialiste » au tournant du siècle. Mais si cette périodisation revêt un caractère systématique, son caractère plaqué est évident: à l'exception d'I. G. Saouchkine (1976), aucun auteur n'essaie véritablement d'ancrer la production géographique du XIXe siècle dans le contexte d'un essor capitaliste et/ou impérialiste. Aussi ne faut-il pas accorder trop d'importance à cette grille historique; le maillage en est fort lâche et la matière évoquée s'épanche souvent hors du cadre qui lui est fixé.

\footnotetext{
${ }^{3}$ Cette production a été inventoriée lors d'un séjour de deux mois à Moscou en janvier-mars 1992. L'inventaire englobe le fonds de la Bibliothèque publique et historique de Moscou et celui de la Bibliothèque de l'Institut de Géographie de Moscou. Sans doute est-il incomplet, eu égard aux caractéristiques de la production universitaire soviétique, qui était fort morcelée, voire parfois cloisonnée.

${ }^{4}$ Nous ne comptabilisons ici que les écrits consacrés par exclusive à l'histoire de la géographie russe.

${ }^{5}$ Bogdanov V. V. (ss. la direction de), D.N. Anoutchin, Sbornik v tchiest' 70 liétiïa profiessora D.N. Anoutchina (D. N. Anoutchine. Recueil pour les 70 ans du professeur Anoutchine), Moscou, Éditions de l'Association des Amateurs de Sciences Naturelles, d'Anthropologie et d'Ethnologie, 1913, p. VIII-XL.

${ }^{6}$ Berg, L. S., Otcherk istorii guiéografitcheskoï naouki — vplot' do 1923 (Aspects de l'histoire de la science géographique - jusqu'en 1923), Leningrad, Éditions de l'Académie des sciences de l'U.R.S.S. (EASU), 1923. Berg, L. S., Otcherki po istorii rousskikh guiéografitcheskikeh otkrytiï (Aspects de l'histoire des découvertes géographiques russes), Moscou, EASU, 1949, 254 p.
} 
Au vu de ces premiers éléments de présentation, on entrevoit déjà pour partie ce qui distingue cette historiographie disciplinaire soviétique : elle n'est pas le fruit d'une épistémologie de combat mais, bien au contraire, un moyen d'asseoir et d'uniformiser les représentations de la discipline. Notre hypothèse est qu'il s'agit d'élaborer un credo officiel destiné aux étudiants, avec pour horizon la normalisation des pratiques de recherche.

\section{Finalités et idiosyncrasies d'un genre scolaire}

L'absence presque systématique de paratexte ${ }^{7}$ rend difficile la recherche des destinataires de cette historiographie. Cependant, quelques indices sont éclairants. Le ton employé, didactique dans de très nombreux cas, la très faible technicité du propos, le recours systématique aux définitions pour éclairer le lexique de base de la discipline, enfin l'organisation très simple du discours, tous ces éléments accréditent l'idée d'un genre scolaire. La prédominance des livres sur les articles renforce cette impression. Enfin, environ 20\% du fonds étudié affiche clairement son genre : «leçons » (liektsii, konspiek. liektsii, etc.), «méthodologies », otcherki $\imath^{8}$, etc.

Cette finalité scolaire posée comme hypothèse, on comprend beaucoup mieux le caractère compilatoire et répétitif de nombre de ces ouvrages : leur objectif n'est pas de produire une pensée neuve, originale, mais de cristalliser une représentation de l'histoire de la discipline dans la perspective d'un consensus de la communauté géographique. À ce titre, il est intéressant de s'interroger sur un lieu commun extraordinairement tenace de l'épistémologie soviétique, héritière en cela de ses précurseurs russes : il n'existe pas de limite méthodologique et disciplinaire entre l'exploration géographique et la science "géographie ». Ainsi, le célèbre explorateur Prjévalskiï (1839-1888) prend place dans les manuels au même titre que les principaux géographes savants, P. P. Sémionov "Tian Chanskiï» (1825-1914) et D. N. Anoutchine (1843-1921). Cette articulation très forte entre l'exploratoire et le scientifique, présente dans toutes les écoles nationales, prend une dimension particulière, s'agissant de la Russie, impériale tsariste ou impériale soviétique. Dès sa fondation en 1845, la très officielle Société impériale russe de géographie (abréviation russe: IRGO), a été investie d'une mission de patronage et de systématisation des explorations sur les marges de l'Empire (Sibérie, Asie centrale, frontière chinoise). Après 1918, le relais a été pris par la section de géographie de l'Académie des sciences de l'URSS. Dans un pays aussi vaste et mal-connu, où le pouvoir autoritaire est menacé de dilution sur ses marges, s'est toujours maintenue une forte demande politique de devisement intelligible et systématique du territoire. Les «géographes » et les "militaires », deux catégories souvent confondues, ont été les agents principaux d'une appropriation intellectuelle de l'espace. À ce titre, Prjévalskii est l'archétype de l'arpenteur-deviseur assumant tout à la fois des fonctions d'autorité (militaire) et de recensement (les récits de voyage qu'il a laissés étaient accompagnés de volumineuses tables statistiques). La fonction exploratoire devenait dès lors consubstantielle à la géographie russe: "l'imbrication (vzä̈most' sviazl) entre ces deux aspects historiques de la géographie (les découvertes géographiques et le développement de la science géographique) est lié au fait que les découvertes géographiques contribuent au développement de la géographie comme science, exercent une influence des plus sérieuses sur son contenu, tandis que la théorie géographique conditionne les succès dans la sphère des découvertes » a pu écrire l'un de nos historiographes ${ }^{10}$, discours d'évidence que l'on retrouve chez presque tous les auteurs soviétiques.

\footnotetext{
${ }^{7}$ Cf. G. Genette, Seuils, Eds du Seuil, coll. « Poétique », Paris, 1987. Par « paratexte » ou « seuils éditoriaux », on désigne l'ensemble des formulations marginales qui accompagnent un texte : titres, notes, préfaces, citations, quatrièmes de couverture, etc.

8 Il s'agit là d'un genre à part entière, que l'on peut traduire par «Traits » ou « Aspects », très fréquent dans la production russe. Le propos y est délibérément synthétique, non définitif, destiné à un public profane.

${ }_{9}^{9}$ Lequel a d'ailleurs bénéficié de cette appellation spécifique — et ceci de manière très officielle — à la suite de son périple de jeunesse dans le Tian-Chan, épisode qui fonde sa légende dorée de géographe.

10 A.I. Klimov, Istorïa guiéografitcheskikh otkrytï̈ i razvitïa guiéografitcheskö̈ naouki - Kratkï otcherk. (Histoire des découvertes géographiques et du développement de la science géographique), Gorkii, Eds. de l'université Volga-Viatka, 1978, 88 p.
} 
Ce lien très fort a pour corollaire une autre constante de l'historiographie soviétique : sa prédilection pour la géographie physique. En 1989 Roger Brunet déplorait, dans un numéro de l'Espace géographique ${ }^{11}$, la domination écrasante des géographes physiciens en URSS Cette situation n'est pas nouvelle et se retrouve dans l'historiographie disciplinaire. Comment comprendre le phénomène? Il nous semble que la géographie russe a toujours été plus «naturaliste » que la géographie française. Sa vocation exploratrice n'est sans doute pas étrangère à cet état de fait : commanditée par un État omniprésent, indissociable d'une pratique cartographique, faite de nomenclatures et d'inventaires, tournée vers des espaces sauvages et faiblement anthropisés, la géographie russe réclamait des géodésistes, des naturalistes, voire des ethnologues ${ }^{12}$ pour satisfaire la demande socio-politique dont elle était tributaire. Le déterminisme n'est évidemment pas absolu et se complique de paramètres annexes; cependant, cette explication est la plus satisfaisante pour rendre compte du déséquilibre qui s'est creusé entre géographie humaine et géographie physique. Des trois sections principales de l'IRGO, géographie physique, statistique et ethnologie, seule la première s'est effectivement maintenue dans le giron de la géographie en tant que discipline universitaire. Il faut aussi faire la part des stratégies de recherche des géographes «majeurs »: Sémionov, Anoutchine, mais également Vladimir Dokoutchaïev ${ }^{13}$ (1843-1903) et, dans une moindre mesure, Alexandre Voïeïkov (1842-1916). Commanditaires de travaux de recherche à la Société impériale de géographie (Sémionov) ou à l'université de Moscou (Anoutchine), ils ont orienté résolument la géographie russe dans une perspective naturaliste.

Cet aspect premier a été renforcé par les centres d'intérêt des historiographes soviétiques : à de rares exceptions, ceux-ci n'étaient pas des spécialistes mais des sommités de la discipline. Leur intérêt pour le passé était conditionné par leur champ de recherche en géographie, physique dans la majorité des cas. L'exemple de Liov Sémionovitch Berg (1876-1950) est emblématique. Disciple d'Anoutchine et de Dokoutchaïev, fondateur (parmi d'autres) du landchaftoviédiénizié, sommité de la géographie soviétique, L. S. Berg a écrit une série d'ouvrages historiographiques durant les vingt dernières années de sa vie, qui sont une sorte de testament timide, à travers lesquels il distille idées personnelles, souvenirs et vénérations. Géographe physicien, Berg occulte complètement l'apport des anthropogéographes de sa génération ${ }^{14}$. Encore trouve-t-on chez lui un souci de l'exhaustivité biographique qui nous permet de découvrir les diverses facettes de "géographes» aussi composites que le furent Piotr Sémionov Tian Chanskii et Dimitri Nikolaiévitch Anoutchine. Les premiers spécialistes/praticiens de l'histoire de la géographie russe ont émergé dans les années 1960/1970, alors que la discipline connaissait des secousses importantes, à l'exemple du reste du $\mathrm{Monde}^{15}$. On pourrait citer T. P. Matviéiéva, I. G. Saouchkine (rénovateur de la géographie humaine), L. S. Abramov, V. A. Essakov, E. P. Mourzaïev et plus récemment P. M. Poliane. La première génération s'est efforcé de rationaliser et systématiser la notion d'école géographique ${ }^{16}$. La deuxième s'est pour l'instant affirmée à travers des biographies rénovées ${ }^{17}$ (c'est-à-dire moins contraintes dans ce qu'elles pouvaient

${ }^{11}$ R. Brunet, « Géographies soviétiques », L'espace géographique, 1989, n 1, p. 69-72.

12 Il est à ce propos important de souligner le maintien, durant tout le XIX ${ }^{\mathrm{e}}$ siècle, d'une remarquable section d'ethnologie au sein de la Société impériale russe de géographie.

${ }^{13}$ Pédologue, collaborateur de la Société de libre-économie. Les géographes soviétiques l'ont « annexé » à leur discipline au nom de l'influence qu'il avait eue sur eux.

14 Arséniev (1789-1865), Tchikhatchev (1808-1890), Voïeïkov (1842-1916), principaux jalons de la géographie humaine et/ou environnementaliste russe, sont absents des livres de Berg. Véniamine Sémionov (1870-1942), figure de proue de l'anthropogéographie russe présoviétique, a droit à un meilleur traitement : quelques lignes pour rappeler sa vision de la géographie comme « description de pays ».

${ }^{15}$ Sur ce sujet, of. l'article d'A.-L. Sanguin, « La controverse Anoutchine », L'Espace géographique, 1979, nº 4, p. 273-278.

16 Cf. L.S. Abramov, Opissanïa prirody nachë strany. Razvitiü fiziko-guiéografitcheskikh kbaraktiéristike (La description de la nature de notre pays. Le développement des caractérisations physico-géographiques), Moscou, « Mysl' », 1972. L.S Abramov \& V.A. Essakov, «Probliéma naoutchnykh chkol v istorii guiéografii » (Le problème des écoles géographiques dans l'histoire de la géographie), dans Collectif, Les Écoles scientifiques en géographie, Moscou, 1983, 117 p.

${ }_{17}$ Cf. E.P. Mourzaiev, Liov Semionovitch Berg, 1876-1950, (en russe), Moscou, « Naouka », 1983 et P.M. Poliane, Véniamine Petrovich Sémionov Tian-Chanskï (en russe), Moscou, "Naouka », 1989. Ces deux biographies ont fait l'objet d'une fiche de synthèse pour Geographers : Bio-bibliographical Studies, respectivement vol. 5, p.1-7 et vol. 13 (1991), p. 73-79. 
révéler). Malgré cela, la géographie physique reste structurellement dominante comme objet et les analyses politiques et sociales ne font qu'affleurer.

\section{Pauvreté des analyses sociales et politiques}

Pour des raisons d'évidence, l'historiographie soviétique a fait montre pendant plus de soixante ans d'une extraordinaire insipidité sociale et politique. L'analyse «externe $»^{18}$ a été systématiquement évitée et la géographie humaine prérévolutionnaire, plus proche des libéraux et des socialistes révolutionnaires que des bolcheviks, a été largement occultée. Le seul distinguo recevable tend à dissocier les géographes "progressistes", c'est à dire adversaires du déterminisme naturel, des réaktsioniéry. Dans sa recherche de pères fondateurs, l'historiographie soviétique ne pouvait s'appesantir sur les origines sociales et l'activité d'hommes que tout séparait du bolchévisme. Que dire des Sémionov père et fils, issus de la petite noblesse, qui ont été de fidèles serviteurs du régime tsariste au nom de leur nationalisme grand-russe? Que dire de Dimitri Anoutchine, premier professeur d'université en 1884, à l'époque où le sinistre Konstantin Pobiédonotsev ${ }^{19}$ faisait la chasse aux éléments révolutionnaires dans l'université et réformait les programmes dans une perspective très réactionnaire ? Plus largement, l'historiographie soviétique est fort parcimonieuse lorsqu'il s'agit d'établir des liens et de restituer un contexte. Ainsi, il est rare que des filiations géographiques, des cercles d'affinité soient explicitement désignés; l'histoire de la discipline semble procéder d'une succession d'individualités et d'institutions sans liens. Quelles ont été les influences principales de la géographie russe ? Comment interpréter le séjour de Piotr Sémionov à Berlin en 1853-1854, son amitié avec Ritter et sa traduction de Die Erdkunde von Asien ${ }^{20}$ ? Comment justifier de l'annexion de Vladimir Dokoutchaïev (1846-1903) à la géographie, alors que ce pédologue ne s'est jamais considéré comme tel et n'a jamais publié dans une revue de géographie ? Pourquoi Anoutchine (1843-1923), anthropologue et ethnologue de formation, est-il devenu finalement géographe? Voici quelques-unes des questions mystérieuses que pose la lecture des historiographes soviétiques.

Avec eux, tout se passe donc comme si la géographie russe s'était développée ex nibilo, sans apports extérieurs, sans modèles intra- ou extragéographiques, mais aussi sans débats d'idées, sans polémiques. À de rares exceptions ${ }^{21}$, les auteurs soviétiques nous présentent une géographie lisse et sans aspérités, mue par un progrès historique qui la finalise, "positive » en quelque sorte, la Vérité parée des atours du matérialisme. Et lorsque Dokoutchaïev critiqua l'hétérogénéité des critères utilisés par la Société impériale de géographie pour régionaliser la Russie, il commit une bévue, ce que lui reprocherait Berg soixante ans plus tard: "On ne peut pas être d'accord avec Dokoutchaïev. » Pourquoi ? On l'ignore et le devine à la fois : pour une historiographie qui a pour objectif d'établir un consensus normatif autour d'une histoire légitimante, légende dorée de la géographie russe, toute distorsion harmonique est à proscrire. Cette situation épistémologique nous renvoie toujours au problème de l'émergence d'historiens des sciences qui ne soient pas seulement des géographes "débauchés » dans l'historiographie, mais de véritables spécialistes pouvant profiter de l'apport des sciences sociales contemporaines. L'acte d'écrire une histoire de

\footnotetext{
18 À la suite notamment de V. Berdoulay (1981), nous entendons par analyse externe une approche de l'histoire des sciences qui privilégie l'étude du contexte historique et social dans lequel s'est développé une science.

${ }^{19}$ Konstantin Piétrovitch Pobiédonotsev (1827-1907) a été le précepteur d'Alexandre III (1865), avant de devenir procureur du Saint-Synode (ministre du culte) sous le règne d'Alexandre III (1881-1894). Il a exercé une influence considérable sur la politique scolaire et universitaire de l'époque. Ultraconservateur, il a obtenu que les programmes soient refondus au profit de l'enseignement militaire et religieux, jugé moins subversif (sic) que les sciences humaines et les langues vivantes.

${ }^{20}$ Parce qu'il a été l'élève de Ritter, qu'il a traduit ses livres, avant de diriger l'IRGO pendant quarante ans (de 1873 à 1914), des auteurs, tels L.S. Abramov, font de Piotr Piétrovitch Sémionov «Tian Chanskiï» le fondateur d'une école de géographie comparée, sans s'interroger plus avant quant à la légitimité d'une telle appellation. Faire des descriptions itinérantes des voyageurs russes un modèle méthodologique hérité de Ritter nous semble un peu hasardeux.

${ }^{21}$ Cf. infra: I.G. Saouchkine, Istorïa i miétodologuï̈a guiéografitcheskoï naouki (Histoire et méthodologie de la science géographique), M., 1976 et T.P. Matviéiéva, Rousskizé guiéografy i poutiéchtchiestvienniki. Fondy arkbiva G. O.-spravotchnik. (Les géographes et voyageurs russes. Guide du fonds d'archives de l'IRGO), 1992.
} 
la géographie demeure sinon un acte de mémorialiste, initiative individuelle aux motivations ambiguës et la lecture de ces ouvrages laisse un sentiment d'arbitraire assez profond ; une fois l'orthodoxie satisfaite et le consensus respecté, il est peu important de suivre une problématique nette et ferme. Le statut de livre scolaire peut, là encore, expliquer bien des choses.

Il serait toutefois injuste de terminer cette présentation de l'historiographie soviétique sans insister plus en détail sur l'évolution des approches mises en oeuvre.

\section{Trois exemples / deux moments}

À travers trois ouvrages notables de l'historiographie soviétique, nous voudrions indiquer comment s'est opérée la relative professionnalisation du discours en une vingtaine d'années. Le premier de ces livres a été écrit par Liov Sémionovitch Berg à la fin des années 40 : Aspects de l'bistoire des découvertes géographiques russes (1949). Les deux autres, La description de la nature de notre pays. Le développement des caractérisations physico-géographiques (1972) de L. S. Abramov et Histoire et méthodologie de la science géographique (1976) de Iouri Saouchkine appartiennent au mouvement de renouveau des années 1970.

* Liov S. Berg: Aspects de l'bistoire des découvertes géographiques russes (Otcherki po istorii rousskikh guiéografitcheskikh otkrytii), Moscou, EASU, 1949.

Liov Sémionovitch Berg (1876-1950) a été l'un des premiers élèves d'Anoutchine dans les années 1890, avant de devenir l'un des plus éminents géographes de l'après-Révolution, bien que sa carrière ait déjà commencé avant. Les productions de L. S. Berg dans le domaine de l'histoire de la géographie sont emblématiques de ce que l'on pourrait appeler l'historiographie anténormative: l'appareillage marxiste plaqué n'a pas encore diffusé, de sorte que l'auteur semble complètement à l'écart de la phraséologie soviétique. Il procède par portraits. Ses Aspects... ressemblent à un album de famille, où l'anecdote personnelle voisine avec quelques considérations privées sur l'essence de la géographie. L'intérêt majeur du livre réside dans l'abondance de matériaux rapportés: Berg cite abondamment ses maittres (P. P. Sémionov, Dokoutchaïev, Anoutchine), sans quasiment faire de commentaires et la bibliographie est riche d'ouvrages pré-soviétiques qui disparaittront chez ses successeurs. Les Aspects sont très sélectifs et ignorent un certain nombre de géographes russes importants: K. Arséniev, A. Voïeïkov, Véniamine Sémionov, précisément ceux qui avaient essayé d'infléchir la géographie russe vers des problématiques prenant l'homme en compte, et pour les deux derniers, sans doute peu favorables aux bolchéviks.

Le propos de Berg est souvent très anecdotique : sa démarche biographique relève du genre du portrait, sa compréhension de l'action des sociétés savantes se réduit à un catalogue d'expéditions et de travaux, ses réflexions méthodologiques sont imprégnées d'une évidence frustrante. Toutefois, il est le seul historiographe à partir duquel on puisse poser la question de l'origine sociale des savants, étant le seul à raconter leur vie. Ainsi :

Dimitri Nikolaiévitch Anoutchine est né le 27 août (8 septembre) 1843. Son père, Nicolas Vassiliévitch, originaire de la région de Viatka (district de Norilsk), appartenait, par ses origines sociales, à l'Église. À l'origine, il avait étudié au séminaire de Viatka, puis avait fait son service militaire, s'était illustré durant la première Guerre Patriotique ${ }^{22}$ et avait participé à la prise de Paris, en 1814. La mère de Dimitri Nikolaiévitch, Tatiana Firsovna, née Zacharova, fille d'un paysan aisé de la région de Kostroma (district de Galitchskii), avait reçu une formation dans l'une des pensions pour jeunes filles de Saint-Petersbourg. Dimitri Nikolaiévitch était le dernier de six enfants. Sa mère mourut lorsqu'il avait une douzaine d'années, moins d'un an après quoi Dimitri Nikolaiévitch perdit également son père. D'après la nièce de Dimitri Nikolaiévitch, Sofia Mikhailovna, le futur géographe et académicien était, au début de sa scolarité au Gymnasium, un petit garçon appliqué, modeste, timide et peu causant. Sa santé était fragile et sa mère pensait que son préféré ne survivrait point. À l'école, il ne participait jamais aux galopinades et espiègleries de ses camarades, et sa conduite était généralement exemplaire. À son frère Alexandre, alors étudiant (et

\footnotetext{
22 Il s'agit de la campagne de Russie de Napoléon I ${ }^{\text {er }}$ (1812), ainsi désignée en Russie.
} 
mort en 1856), le petit Anoutchine aurait demandé de lui enseigner comment rédiger les « compositions authentiques» (nastoïachtchiiié sotchiniéniiia), comme en écrivent les adultes. Chez les Anoutchine, on ne connaissait pas le besoin (etc.)».

Ainsi se construisent les enfances célèbres et les images d'Épinal.

Le genre développé ici renvoie tout à fait à ces «vies d'hommes remarquables» (jizni zamiétchatiel'nykh lioudiei) qu'affectionnaient les Soviétiques. Au-delà de l'anecdote, on voit bien le profit (mince) que l'on peut tirer de ce type de production : L. S. Berg nous renseigne sur l'origine sociale de Dimitri Anoutchine (1843-1923). Dans un Empire russe où la bourgeoisie était chose rare, sa trajectoire est un exemple d'ascension sociale. Mais si les étudiants provenant de la petite bourgeoisie ecclésiastique et militaire ont fourni les plus gros bataillons de révolutionnaires intraitables à partir du règne d'Alexandre II (1855-1881), on ne trouve rien de tel chez Anoutchine, qui s'est remarquablement intégré dans les milieux universitaires. On s'interrogera d'ailleurs sur l'itinéraire d'un homme que rien n'a déstabilisé, ni le ministère de Pobiédonotsev (1881-1894), ni la révolution d'Octobre ${ }^{23}$. À l'heure actuelle, les engagements effectifs d'Anoutchine demeurent un mystère, que l'historiographie soviétique s'est bien gardée de percer $^{24}$.

Malgré leur abondance et leur vaste postérité, les écrits de Berg ne relèvent pas d'une historiographie concertée. Il n'y a ni réflexion préalable quant à la méthodologie du genre, ni problématisation, ni production d'outils spécifiques. La discipline est dans son état d'enfance, c'est-à-dire dénuée de cette part d'auto-réflexion qui fonde la rationalité des sciences sociales.

*L. S. Abramov : La description de la nature de notre pays. Le développement des caractérisations physico-géographiques (Opissaniiia prirody nacheï strany. Razvitiié fiziko-guiéografitcheskikh kharaktiéristik), Moscou, « Mysl'», 1972.

Ce deuxième ouvrage, paru 23 ans plus tard, nous a été présenté par son auteur lui-même comme la première synthèse soviétique pertinente concernant l'histoire de la géographie russe. Contrairement à ce que le titre laisserait indiquer, il n'est pas seulement question de géographie physique et l'essentiel est repris dans deux tableaux synoptiques dont on trouvera la traduction française ci-jointe. L'élément novateur propre à l'ouvrage d'Abramov réside dans sa volonté d'isoler des écoles de géographie soigneusement définies et différenciées. L'usage du terme «école » était plus que banal dans l'historiographie soviétique au moment de la parution du livre ; la nouveauté tient ici à la déclinaison systématique de l'expression. Auparavant, l'«école géographique » renvoyait à un maitre (M. Lomonossov, Sémionov, Dokoutchaïev, Anoutchine), laissait vaguement entendre une postérité et des disciples, mais il était rare que soient véritablement caractérisés des traits dominants et une démarche ${ }^{25}$. Chez Abramov, le terme prend une plus grande épaisseur. Le tableau $n^{\circ} 2$ montre déjà une collectivisation de la notion. Il n'est plus question de «l'école de Sémionov» ou de «l'école d'Anoutchine». Chacune a plusieurs précurseurs, plusieurs "fondateurs et leaders » et s'inscrit dans une institution particulière (la Société impériale russe de géographie, l'université de Moscou, la Société de libre-économie ${ }^{26}$, etc.).

\footnotetext{
${ }^{23}$ Bien au contraire, puisque la « béatification » du père fondateur s'est faite à l'époque soviétique.

${ }^{24}$ La biographie de D.N. Anoutchine dans Geographers : Bio-bibliographical Studies, vol. 2, p. 1-8, rédigée par Vassiliï Alexiêiévitch Essakov, est très frustrante, car elle ne nous apprend strictement rien de neuf sur la trajectoire et les intérêts d'Anoutchine, si ce n'est qu'il aurait lu dans sa jeunesse Biélinskiï et Herzen, les deux grands fondateurs du socialisme russe.

25 Ainsi, il est souvent question de l'école d'Anoutchine, au vu des travaux de ses disciples, c'est à dire de ses étudiants à l'université de Moscou. Ce raccourci est à la fois tentant et problématique : il faudrait pouvoir prouver qu'Anoutchine a apporté quelque chose de nouveau à la discipline. Or, rien n'est moins sûr: polygraphe étonnant par la diversité de ses intérêts, enseignant, journaliste, Anoutchine a peut être été un accoucheur plutôt qu'un chercheur - innovateur, dans la sphère de la géographie du moins. $C f$. notre 2ème partie, «Une institutionnalisation inachevée ».

${ }^{26}$ Fondée par Catherine II sous l'influence des physiocrates, la Société de libre-économie (Vol'no-ékonomitcheskoüé Obchtchestvo) avait pour mission d'apporter davantage de bien-être au peuple russe par l'étude de l'appareil productif et la proposition de réformes. Très vite, il s'est avéré qu'elle n'avait aucun pouvoir d'influence sur l'Impératrice. Elle s'est alors commuée en une société savante d'inspiration libérale modérée. À la fin du XIXe siècle, elle comptait nombre de savants et d'intellectuels prestigieux dans ses rangs (dont Dokoutchaïev et Voïeïkov). L'économie politique et l'agronomie furent ses principaux chevaux de bataille.
} 
Abramov prend soin de définir un objet d'étude, de détailler la méthodologie et de préciser les principales productions de l'école. Plutôt que de commenter l'ensemble de l'ouvrage, indisponible en français, attachons-nous aux deux tableaux synoptiques ci-joints, qui nous permettront d'éclairer l'intérêt et les limites de la démarche d'Abramov. La présence de ces documents indique déjà une nouveauté par rapport aux histoires de la géographie antérieures, uniquement textuelles.

Le tableau $\mathbf{n}^{\mathbf{0}} 1$ essaie de matérialiser la période d'activité de la plupart des écoles définies par Abramov. Le formalisme employé (basé sur des cartouches verticaux) laisse entendre que toute école a une origine diffuse (symbolisme du trait linéaire), une période d'affirmation (take off? le trait s'évase en surface), une période de plein développement (la bande du cartouche occupe une largeur maximale), puis décline et disparait, laissant parfois un vague héritage (retour au trait linéaire). Autour du graphe, l'auteur a fait figurer un certain nombre d'indications. Les écoles géographiques sont désignées par une appellation précise, qui figure au-dessus de leur représentation en cartouches temporels : "école de géographie statistique », "école de géomorphologie », "école soviétique de géographie physique », etc. S'agissant des trois premières écoles russes, Abramov souligne qu'elles n'ont pas eu le temps de se développer pleinement. Horizontalement, l'auteur précise un certain nombre de données historiques et historiographiques. Ainsi, on retrouve en colonne 1 la vulgate chronologique que nous avons évoquée précédemment. Plus curieuse est la deuxième colonne du tableau, qui décrit des "périodes d'étude géographique de la nature du pays», concept inédit qui redouble l'organisation verticale. D'une certaine manière, cette seconde délimitation historique implique un principe d'enchaînement entre des phases qui s'excluent chronologiquement. Or, le reste du tableau, c'est-à-dire le graphe lui-même, est fondé sur l'idée d'une cohabitation entre des écoles différentes. On peut se poser beaucoup de questions sur l'effet obtenu : la précision historiographique enrichit-elle la compréhension du graphe, ou, au contraire, n'introduit-elle pas une discordance méthodologique ? Et que dire de l'association de deux grilles historiques hétérogènes et de deux conceptions historiographiques contradictoires? Ces contradictions sont occultées par la netteté du découpage chronologique, dont la rigueur serait à discuter.

La règle d'allure logarithmique employée pour figurer le temps est productrice d'une incidence de sens qu'il est intéressant de mentionner. En effet, on constate que les 55 ans qui séparent la révolution d'Octobre de l'année 1972 sont munis d'une longueur de cartouche supérieure à celle qui représente les 217 années précédentes (1700-1917). Une disproportion existe également entre le XVIII ${ }^{\mathrm{e}}$ et le XIX ${ }^{\mathrm{e}}$ siècle. Serait-ce l'avènement de la scientificité et/ou de la complexité ? L'Union Soviétique aurait-elle connu de très nombreuses fondations d'écoles ? À vrai dire, le tableau d'Abramov indique exactement le contraire: les deux écoles nées sous le régime soviétique étaient déjà bien établies en 1930 (après l'instauration du stalinisme comme système politique). L'utilisation d'une règle d'allure logarithmique ne se justifie donc pas du point de vue historique. Le sens est ailleurs. Est-il besoin de préciser que l'allongement des quatre derniers cartouches est démonstratif de leur postérité éminente? Métaphoriquement, le graphe nous fait voir les quatre piliers du temple de la géographie soviétique, c'est à dire les quatre écoles de géographie « matérialistes » russes.

Le tableau $\mathbf{n}^{\mathbf{0}} \mathbf{2}$ complète le premier en précisant les institutions, les lieux, les hommes, leur méthodologie et leur passé. Chaque école occupe cette fois un niveau horizontal, le sens de l'histoire étant donné verticalement, de haut en bas. Problème majeur pour qui ne connait pas la géographie russe : les ouvrages cités dans la sixième colonne le sont sans mention des auteurs ${ }^{27}$. Plusieurs remarques s'imposent à la lecture de ce tableau.

\footnotetext{
${ }^{27}$ Nous avons précisé que Le relief superficiel de la plaine de Russie (1895) était d'Anoutchine. Le Tchernoziom russe (1886) est le livre majeur de Dokoutchaïev. La Russie pittoresque (1882) est un ouvrage de P. P. Sémionov destiné au grand public et La Russie. Description complète de notre patrie (1889-1914) est une collection d'ouvrages de géographie régionale qu'a dirigée le fils de P. P.
} 
* Certains géographes se retrouvent à la fois en aval de certaines écoles et en amont d'autres. Ainsi, Prjéval'skiii serait un continuateur de l'école des officiers d'état-major et l'un des leaders de l'école de l'IRGO. Louable intention que d'établir des continuités! Surtout entre une école de géographie physique et une école de géographie humaine...

* La question de la distinction entre géographies physique et humaine n'est pas explicitée ici, mais comme les écoles sont les mêmes que dans le tableau $\mathrm{n}^{\circ} 1$, on peut sans doute appliquer le distinguo au tableau n ${ }^{\circ}$ 2. Dès lors se pose la question de savoir comment Liov Abramov arrive à faire des Sémionov père et fils de purs géographes physiciens? L'activité de P. P. Sémionov jusque dans les années 1870 le justifierait peut-être. Mais par la suite, celui qui fut vice-président de la Société impériale de géographie n'a plus guère produit dans ce domaine, se consacrant avant tout à son grand oeuvre de recensement de la population impériale et à des travaux de régionalisation du territoire russe. Dans le cas de son fils, la contradiction nous apparait encore plus flagrante ( $c f$. note 14). Pourquoi également associer dans la même rubrique des « descriptions de voyageurs» qui sont un genre en soi et les ouvrages de vulgarisation, non exclusivement "physiciens» (loin s'en faut) que sont le Dictionnaire géographique et La Russie pittoresque de P. P. Sémionov et La Russie. Description complète de notre patrie? Ici, Abramov mélange peut être un peu hâtivement les genres.

* Généralement, les fondateurs et leaders d'une école sont de la même génération. À ce titre, la rigueur la plus élémentaire aurait voulu que Véniamine Sémionov apparaisse parmi les «Représentants des générations ultérieures» de l'École géographique de description comparée, alors qu'il côtoie ici son père et Prjéval'skiï. Ceci est d'autant plus étrange qu'il est difficile d'évaluer ce qui lie ce dernier, militaire, ornithologue et naturaliste à V. P. Sémionov, fondateur de la géographie urbaine russe. D'autres fantaisies rendent surprenante la lecture de ce tableau : l'article d'Anoutchine qui est cité est essentiellement historiographique et aurait du mal à fonder la géomorphologie russe. Et l'on est surpris de voir figurer le grand publiciste russe Alexandre Herzen parmi les précurseurs de la géomorphologie.

Ces deux tableaux de L. Abramov sont tout à la fois intéressants par la tentative qu'ils manifestent et discutables du point de vue de la rigueur. Faut-il y voir une faiblesse inhérente à la notion d'école, qui confond le contenant avec le contenu ? Faut-il se plaindre de la légèreté avec laquelle l'auteur crée des écoles: pas moins de neuf pour la seule Russie en moins de deux siècles ? L'absence de référence aux principales grilles épistémologiques contemporaines et une conception très lâche de ce qu'est une école scientifique sont peut-être les principaux handicaps du travail d'Abramov, outre la rapidité d'exécution de l'ouvrage (deux mois), qui nous a été confessée par l'auteur. Pour le reste, le livre proprement dit comporte des pages très éclairantes sur le développement de la statistique dans la Russie tsariste et a le mérite essentiel d'établir une classification de la géographie russe prérévolutionnaire, fût-ce au prix de quelques légèretés. Il s'inscrit ainsi comme le premier jalon de la professionnalisation de l'historiographie russe, professionnalisation inachevée du fait de l'absence de toute référence externe, mais indubitable.

* Iouri Saouchkine : Histoire et méthodologie de la science géographique (Istoriïa i miétodologuiïa guiéografitcheskoï naouki), M., 1976.

Cet ouvrage de $1976^{28}$ est la transcription de cours professés par Iouri Saouchkine à l'université de Moscou. Mais l'aspect pédagogique n'est pas handicapant : à rebours de toute l'historiographie soviétique d'alors, ce livre est traversé par une problématique forte, par un

Sémionov, Véniamine Piétrovitch, anthropogéographe (donc « ratzélien »?), auquel on doit bon nombre d'ouvrages de géographie humaine.

${ }_{28}$ En 1976, année faste, ont été publiés deux ouvrages collectifs en anglais: History of Geographical Thought, Abstract of papers, Leningrad, Geographical Union, ouvrage plus ou moins initié par T.P. Matviéiéva et A Short History of Geographical Science In The Soviet Union, Progress Publishers, Moscow, publié sous la houlette du très académique I. Guérassimov. Si le premier de ces deux livres consacre l'émergence de véritables professionnels de l'histoire de la géographie, le second est une sorte de quintessence du soviétisme historiographique. 
combat. Il s'agit, pour ce spécialiste de géographie économique, de reposer la question de la place de la géographie dans le système universitaire. Principal soutien de Vsiévolod Anoutchine au moment de la fameuse controverse qui secoua la géographie soviétique entre 1961 et $1965^{29}$, Iouri Saouchkine milite pour une géographie unitaire, transversale, recentrée sur les sociétés humaines et l'interface homme/nature, ni déterministe, ni prométhéenne. Parallèlement, le problème de la scientificité de la géographie est posé, qui nécessite une redéfinition des pratiques de recherche, notamment en géographie humaine. La date de parution de cet ouvrage ne doit rien au hasard : elle s'inscrit dans un mouvement de contestation des pratiques de la discipline au nom de ce que doit être une science, qui précède ou coïncide avec la traduction en russe des livres de R. Chorley \& P. Haggett, Les modèles en géographie (1971) et D. Harvey, L'explication en géographie $(1974)^{30}$. Le livre de Saouchkine est d'ailleurs alimenté de très nombreuses références aux géographies occidentales, ainsi qu'à l'historiographie américaine ${ }^{31}$.

L'ouvrage embrasse la totalité de l'histoire de la géographie, mais fait un sort particulier au $\mathrm{XIX}^{\mathrm{e}}$ siècle. Outre son intérêt polémique, il a le mérite d'établir de nombreuses connexions entre la géographie russe et ses consoeurs européennes. L'influence de Ritter et Humboldt sur la génération de Piotr Sémionov est abordée, mais c'est surtout dans l'analyse des débats concernant le rapport des sociétés humaines à la Nature que Saouchkine se révèle intéressant. Il montre clairement l'influence des géographes américains Georges P. Marsh et Arnold Guyot sur certains géographes russes et la fracture idéologique qui s'est créée entre les «optimistes », visionnaires d'une nature aménagée (ou détériorée...) par l'homme, et les réaktsionniéry, pour lesquels celui-ci n'est qu'un élément de celle-là, et se trouve donc conditionné par elle. C'est ainsi que A. Voïeïkov (1842-1916) vient s'inscrire pour Saouchkine dans la tradition du socialisme russe, dans le sillage de L. Mentchikov ${ }^{32}$ (1838-1888), N. $\operatorname{Ogarev}^{33}$ (1813-1876) et P. Kropotkine (1842-1921), à travers ses travaux et réflexions relatifs à l'influence de l'homme sur la Nature. I. Saouchkine est, à notre connaissance, le seul historiographe qui ait accordé une telle place à ce géographe, fort bien connu de ses contemporains français ${ }^{34}$, mais si souvent négligé dans la production soviétique $^{35}$. Sur bien d'autres aspects, il ébauche ce qui manque cruellement à d'autres: la confrontation des traditions nationales, l'ouverture sur des problématiques extragéographiques, etc. On peut seulement regretter que l'auteur n'ait pas pu aller plus loin dans l'éclaircissement de certaines filiations et que l'histoire soit quelque peu absente de la scène géographique, où hommes et idées viennent dialoguer sur des tréteaux soigneusement vidés.

De tous les historiographes rencontrés, I. G. Saouchkine est le seul à assumer un marxisme soviétique qui ne soit pas pure clause de style. La plupart des distinguos établis (géographes progressistes/ géographes réactionnaires, etc. ) le sont à l'aune d'une grille marxiste-léniniste revisitée. Ceci n'empêche pas l'auteur de consacrer de nombreuses pages à $\mathrm{K}$. Ritter et à sa "théologie isotopique », Ritter pour lequel il ne cache pas son admiration, dans sa tentative pour fonder une géographie modélisante. Ici, l'historiographie dit clairement son combat pour la

\footnotetext{
${ }^{29}$ Cf. l'article op. cit. d'André-Louis Sanguin sur « La controverse Anoutchine » dans L'Espace géographique, nº 4, 1979, p. $273-278$.

${ }^{30}$ La traduction russe s'intitule L'explication scientifique en géographie.

${ }^{31}$ Saouchkine cite abondamment l'ouvrage classique de M. Jeffrey \& J. Preston, All Possible Worlds, New York, 1972.

32 Collaborateur d'Elisée Reclus lors de la rédaction de La Terre et les Hommes, membre de l'Internationale socialiste et rédacteur au Kolokol' (la Cloche), le célèbre journal des émigrés russes (1857-1868), dirigé par Alexandre Herzen.

33 Principal collaborateur d'Herzen au Kolokol, publiciste, poète épouvantable, Ogarev (on prononce "agariov») est cité dans nombre d'histoires de la géographie russe comme ayant été géographe à ses heures perdues. Saouchkine lui consacre d'ailleurs quelques pages. Il est inutile de préciser l'effet très valorisant que pouvait avoir l'intégration des grands révolutionnaires parmi les géographes du siècle dernier dans un manuel soviétique en 1976 !

34 Il a publié neuf articles et notes dans les Annales de géographie et nous apparait aujourd'hui comme le géographe russe le plus proche des vidaliens.

35 Nous n'avons que des hypothèses pour expliquer cette négligence : faut-il y voir le reflet d'un problème politique (Voïeikkov n'est jamais passé de l'idéalisme bourgeois au marxisme) ou d'une absence de position institutionnelle dans la discipline " géographie »? Mais l'essentiel est peut être ailleurs : Voïeikov fut un écologiste avant l'heure, l'un des premiers à s'être soucié des effets désastreux de l'action humaine sur la nature. Or, cette position est incompatible avec le messianisme stalinien en vigueur dans la géographie russe jusqu'au début des années 1960 (Cf. à ce sujet l'article d'André-Louis Sanguin op. cit. en note 15).
} 
définition de nouvelles perspectives de recherche, l'évocation du passé visant à influer sur le présent. De la même façon qu'en France, la réhabilitation d'Élisée Reclus s'est effectuée un peu «contre» Vidal de la Blache, dans la Russie soviétique le «retour aux origines » signifie un réexamen de l'apport des pères fondateurs, en rehaussant certains (Ritter, Voïeïkov), en minorant d'autres (Anoutchine et surtout Dokoutchaïev).

Critique, révisionniste, l'ouvrage de Saouchkine a les défauts de ses qualités : ses grilles de lecture sont parfois manichéennes, lancinantes souvent. Le recours à la terminologie soviétique paraît souvent décalé. La convocation de toutes les grandes figures du Socialisme Russe auprès de la Géographie ressemble à un excès de zèle. Cependant, malgré ses insuffisances, il est des plus stimulants et suscite de nombreuses ouvertures historiques et épistémologiques. Il représente un jalon supplémentaire dans le processus de professionnalisation de l'historiographie russe.

Au terme de cet examen, ce qui nous semble le plus décevant dans cette abondante production est sa paradoxale anhistoricité. Par delà les déclarations de principe et la langue de bois, ce qui nous est proposé témoigne d'une sérieuse méconnaissance de l'histoire russe et d'un refus prudent de tout examen en profondeur. Pour advenir comme modèle, le passé se doit d'être dégrossi, épuré de sa contingence, commué en un pur enchaînement d'idées.

Les pages qui suivent ont été conçues en réaction contre l'atonie de l'analyse sociohistorique soviétique. Il s'agit d'ébaucher une autre lecture de l'histoire de la géographie russe, à l'aune des problématiques de l'histoire et de la sociologie des sciences occidentales. Toutefois, il n'est pas question de construire un tableau de la géographie russe au XIX ${ }^{\mathrm{e}}$ siècle, entreprise qui dépasse largement le cadre des recherches menées. Il s'agit plutôt de réemployer la matière soviétique en la confrontant aux quelques acquis occidentaux sur la question, en relation avec le contexte historique russe global tel qu'il a été pensé par les historiens de la Russie pré-soviétique.

\section{Prolégomènes à une autre histoire de la géographie russe au XIX ${ }^{\mathrm{e}}$ siècle}

Aux antipodes d'une synthèse définitive, les pages qui suivent voudraient proposer quelques ouvertures, quelques pistes de recherche, jusqu'ici peu exploitées. Il ne s'agit donc que d'un canevas d'analyse, canevas hérissé de questions sans réponses. La partition choisie est un hybride de plans thématique et chronologique. Il s'agirait de montrer que la géographie s'est développée en Russie en réponse à une très forte demande étatique, de sorte que la cristallisation disciplinaire s'est effectuée dans des cadres institutionnels étroitement liés à l'administration. Avec la lente émergence d'une société civile s'est fait jour une mode de la géographie, source de quelques débats dans l'Intelligentsia. Progressivement, la discipline s'est ainsi sécularisée, de manière plus nette à partir de 1884, lorsqu'un processus d'institutionnalisation via l'université s'est enclenché.

\section{D'une géographie d'officiers à une géographie officielle}

La géographie russe n'a connu qu'en 1845 sa première cristallisation disciplinaire durable, avec la fondation de la Société impériale russe de géographie (Impiératorskoïé Rousskoöé Guiéografitcheskoüé Obchtchestvo ou IRGO) Avant cela ont existé des pratiques diverses que les géographes russes ont annexées à leur discipline, dans un processus classique de constitution d'un passé a posteriori. Il a fallu attendre la fin du XIX ${ }^{e}$ siècle pour que soit ouverte la première véritable chaire de géographie russe. Auparavant, il n'y avait jamais eu de demande véritable dans ce sens. Quelques naturalistes et penseurs allemands (Rost, Diltey, Reichel) avaient exercé au XVIII siècle, dans le cadre de la toute neuve université de Moscou, fondée en avril 1755 par le poète, grammairien et savant Mikhail Vassiliévitch Lomonossov (1711-1765). En 1776 à Saint- 
Petersbourg, des cours étaient professés par un dénommé Chariton Andréiévitch Tchébotarev, tandis qu'en 1804, l'université de la capitale ouvrait deux chaires, l'une consacrée à l'histoire, à la «statistique » et à la géographie du Monde entier, l'autre dévolue, pour les mêmes disciplines, à l'étude de la seule Russie. En 1835, la géographie a disparu, comme discipline, de l'université russe $^{36}$. Cette instabilité va de pair avec une extrême instabilité de l'objet "géographie », dont l'acception variait d'un enseignant à l'autre.

La plupart des historiographes soviétiques ont négligé cette prime géographie universitaire, considérant implicitement que le développement principal de la discipline durant cette période s'était fait ailleurs. Entre 1755 et 1845 ont effectivement émergé des pratiques géographiques extra-universitaires qui allaient conditionner largement le devenir de la discipline. Les règnes d'Elisabeth Pétrovna (1741-1762) et Catherine II (1762-1796) ont été marqués par une amplification du projet impérial russe, systématisant la politique menée par Pierre-le-Grand au début du siècle : il s'agissait de favoriser à long terme la connaissance de la totalité de l'Empire, terra incognita en ses immenses périphéries orientale et méridionale. La production d'un savoir relatif à des territoires méconnus, marginaux et souvent disputés ${ }^{37}$ est constitutive de leur appropriation définitive et de leur intégration. L'acte premier de cette appropriation du territoire fut un inventaire, réalisé par les explorateurs, accompagné ou suivi de travaux géodésiques et cartographiques. Cet inventaire comportait une dimension ethnographique non négligeable, puisque les explorateurs mandatés par les Empereurs rencontrèrent sur leur route les très diverses souches autochtones qui peuplent la Sibérie, le Caucase et l'Asie Centrale, d'où une mission supplémentaire de recensement de ces populations, auxquelles allaient s'adjoindre rapidement les premiers colons et des serfs en fuite. Pour réaliser cet inventaire du territoire, le pouvoir impérial a eu recours à deux types d'agents : les officiers d'Etat-major et les naturalistes, généralement d'origine étrangère tant que l'université russe ne fut pas en mesure de fournir des contingents nationaux suffisants.

S'agissant du premier groupe, il faut insister sur le rôle fondateur de M. Lomonossov ${ }^{38}$, qui a posé les bases d'une méthodologie de recueil d'informations, à visée encyclopédique. Ultérieurement, le relais a été pris par les ministères intéressés à une meilleure connaissance de l'Empire : ministères de la Guerre, de l'Intérieur et des Domaines ${ }^{39}$. Cette « école de statistique », que les géographes russes puis soviétiques ont assimilé à la géographie, a développé des caractéristiques très typées: prédominance du chiffre sur la lettre, esprit de nomenclature, sécheresse et exiguité des commentaires, qui manifestent leur origine militaire et/ou bureaucratique. Ces annuaires statistiques régionalisés ont été très souvent critiqués : par les contemporains (par Konstantin Arseniev notamment), puis par les publicistes radicaux (Vissarion Biélinskiï, Nicolaï Ogarëv, Nikolaï Tchernychevskiii), qui, d'après I. Saouchkine, n'ont eu de cesse de vilipender l'accumulation de chiffres, l'esprit de nomenclature et la stérilité cognitive de cette statistique.

Le deuxième groupe intègre une nébuleuse de naturalistes aux spécialités les plus diverses. L'Académie des sciences a commandité un très grand nombre d'expéditions et de travaux, depuis sa fondation, en 1725, jusqu'en 1845. Il s'agissait d'explorer et de cartographier les marges de l'Empire et, en second lieu, de constituer le territoire en objet scientifique, étudiable par les principales disciplines géographiques (géodésie, topographie, cartographie) et naturalistes

\footnotetext{
36 Sur cette question des chaires de géographie, cf. A. Soloviev, « Kafiédra guiéografii Moskovskovo Ouniviersitiéta s 1917 goda » (La chaire de géographie de l'université de Moscou depuis 1917), Ann.de la Section de Géogr. de l'univ. de Moscou, Fasc. LV, 1940, p. 59. 37 à la Perse, à la Chine, aux Etats-Unis et plus tard au Japon.

38 Abramov (1972) met en scène trois écoles de géographie humaine, qui seraient apparues entre la fin du XVIII siècle et 1830 . Il les appelle "École de statistique camérale ", "École de géographie statistique » et "Descriptions de gouvernements de l'Etat-Major». Il nous semblerait historiquement plus approprié de fédérer ces trois écoles au nom de leur inscription dans le sillage de la volonté impériale et de leur unité méthodologique, héritée de M. Lomonossov.

39 Créé en 1837, le ministère des Domaines était le ministère de tutelle des paysans d'Etat. À ce titre, il était très demandeur d'informations relatives au peuplement et aux mouvements migratoires sur l'ensemble du territoire impérial. Les «Descriptions statistiques de gouvernement » réalisées au XIXe siècle sont le résultat de cette demande ministérielle ( $c$. pages suivantes).
} 
(géologie, botanique, zoologie). La plupart des savants mandatés étaient donc des étrangers, généralement des Allemands ou des Baltes à partir du règne de Paul I ${ }^{\text {er }}$ (1796-1801). On peut citer (entre autres) Euler et De l'Isle, requis par l'impératrice Anna Ivanovna (1730-1740) pour développer la cartographie russe, Pallas, Steller, Georgi, sous le règne de Catherine II, figures emblématiques des outchionyië-poutiéchtchestvienniki, les «savants-voyageurs", chargés de recueillir force informations sur la nature et les peuples de l'empire. Au XIXe siècle, les missions se sont multipliées, exploratrices sur les marges, plus nettement scientifiques au coeur de la Russie. Nombreux sont les savants, et notamment F. Lütke (1797-1882), K. Baer (1792-1876), V. Struve (1793-1864), I. Krusenstern (1770-1846), qui ont été amenés à jouer un rôle d'animation au sein de l'Académie impériale des sciences. La fondation, en 1845, de la Société impériale russe de géographie procède des initiatives de ces explorateurs-naturalistes-ethnologues.

La cristallisation disciplinaire de la géographie russe dans la seconde moitié du XIXe siècle doit beaucoup au double héritage de la «statistique » et du «naturalisme ». Le parallèle avec la France est à ce titre très éclairant. Le façonnement de la géographie française dans les années 1870-1900 s'est opéré à partir de et contre l'histoire, alors que les deux disciplines demeuraient étroitement imbriquées dans l'enseignement. À l'inverse, l'histoire et la géographie russes se sont développées au XIX ${ }^{\mathrm{e}}$ siècle de manière autonome, sans le moindre lien. Discipline résolument naturaliste dans ses manifestations savantes, inventaire statistique pour ses commanditaires officiels, la géographie en Russie est "née » sous des auspices nettement pragmatiques. Recouvrant une grande variété de pratiques, projet encyclopédique de connaissance d'un territoire, celui de l'Empire russe, elle offrait un «nom» et un ancrage savant au projet impérial russe. L'émergence de la Société impériale russe de géographie allait confirmer cette tendance et enraciner le projet géographique dans le nationalisme russe.

\section{Affirmation de la Société impériale russe de géographie}

La fondation de l'IRGO en 1845 procède d'une dynamique tout à fait particulière. La fondation de sociétés de géographie à Londres et à Paris avait été le fait de la société civile, indépendamment du pouvoir en place, et les activités géographiques s'y étaient développées hors du contrôle de l'état. En Russie, il en a été tout autrement. Dans son article "Aux origines de l'ethnographie russe: la société de géographie dans les années 1840-1850 ${ }^{40}$, Wladimir Berelowitch écrit: "Rappelons tout d'abord les circonstances de la création de la société. En 1845, un groupe de Pétersbourgeois, officiers de l'état-major, membres de l'Académie des sciences (géographes et zoologistes), professeurs de l'université de la capitale et fonctionnaires du ministère de l'intérieur, fondent la société de géographie, avec la bénédiction du Comte Ouvarov (ministre de l'Instruction et créateur de la doctrine nationaliste officielle) et de l'Empereur. » Ceci indique très clairement le vivier dans lequel la toute jeune IRGO a puisé ses fondateurs : savants (étrangers bien souvent) et hommes d'état, souvent très haut placés ${ }^{41}$. Pour toute une partie de l'administration impériale, la géographie était donc le réceptacle d'un projet, dont il convient d'esquisser le contenu.

Très logiquement, l'IRGO a dès sa création pris le relais de l'Académie des sciences pour tout ce qui relevait de l'organisation et du financement de voyages d'exploration sur le territoire impérial, ainsi qu'à l'étranger. La première expédition qu'elle ait commanditée s'est effectuée dans l'Oural, en 1847, sous la direction du géologue allemand Hoffman. Après cette date, les expéditions se sont multipliées. Il faudrait mentionner celles de Baer et Danilievskiï dans la région de la mer Caspienne (1853-1857), celles de P. P. Sémionov dans le Tian Chan (1856-1857), de Prjéval'skii dans l'Oussouri (1867-1869) et bien d'autres encore (dont Potanine, Pertsov, Groum-

\footnotetext{
${ }^{40}$ Dans Cabiers du monde russe et soviétique, XXXI (2-3), avril-septembre 1990, p. 265-274.

${ }^{41}$ On compte parmi ses fondateurs Nikolaï Milioutine, futur ministre « libéral » d'Alexandre II. Le président et inspirateur officiel de la Société était le grand-duc Constantin, frère de l'empereur Nicolas I ${ }^{e r}$.
} 
Grimailo, Voïeïkov, etc.). P. P. Sémionov ${ }^{42}$ et plus tard L. S. Berg ${ }^{43}$ ont fait l'historique de cette mission d'exploration du territoire impérial, mais il n'existe à notre connaissance aucun ouvrage analytique qui se soit focalisé sur la problématique coloniale qui sous-tend toutes ces entreprises. Très récemment, Mark Bassin a entrepris de décrypter le nationalisme sous-jacent à tous ces voyages d'exploration dans son très intéressant article, «Russian Geographers and the 'National Mission' in the Far East ». Il écrit ainsi :

(...) it quickly becomes apparent that the process of 'exploration' or 'discovery' in which they were engaged was complex and multifaceted; indeed, it might be said that not one but at least two quite discrete processes were talking places. The first, and more immediately apparent, of these we have just summarized and corresponds to what is traditionally understood by the notion of discovery: navigating and describing unknown waters, surveying coastlines, establishing navigational conditions and so on. The second process was more subtle and corresponded not so much to the need for 'objective' geographical informations as to the needs of the new nationalist consciousness. ${ }^{44}$

Et l'auteur de montrer à travers de nombreux cas (Neviel'skoï, Prjéval'skii,, Sémionov) la manifestation d'une idéologie nationaliste qui pose la Russie en civilisatrice de l'Extrême-Orient barbare. Le colonialisme russe trouve là sa justification et laisse transparaître un deuxième aspect, nettement anti-occidental : des auteurs comme Sémionov insistent sur le caractère beaucoup plus pacifique de la «civilisation» russe, qui n'annihile pas les tribus «à moitié sanvages» mais les intègre graduellement, à l'inverse de ce qui s'est passé en Amérique.

Replacé dans son contexte historique global, ce nationalisme de la géographie russe prend toute sa dimension. À la fin des années 1830, deux mouvements d'opinion ont émergé et polarisé l'embryonnaire intelligentsia russe : slavophiles, partisans d'une régénération de la Société russe par un retour aux sources de la slavitude et occidentaux (ou plutôt : occidentophiles, rapadniki en russe), marqués par la philosophie hégelienne, qui appelaient de leurs voeux une modernisation du pays inspirée par les modèles occidentaux. Les slavophiles ont rencontré un grand succès dans l'aristocratie ${ }^{45}$, séduite par l'esprit nationaliste et «agrarien » de leurs thèses. À la même époque, le comte Ouvarov avait théorisé l'idéologie nationaliste officielle, sorte de récupération du nationalisme au profit de l'autocratie. Aussi, tout indique que les membres russes de l'IRGO étaient imprégnés par le slavophilisme ambiant et qu'ils ont été les propagateurs d'un nationalisme mâtiné de raison d'Etat. Toutefois, la Société a été dominée par les naturalistes allemands (Baer, Lütke, etc.) jusque dans les années 1860, de sorte que ce nationalisme virulent n'est véritablement devenu doctrine qu'avec l'arrivée au « pouvoir» de Piotr Pétrovitch Sémionov «Tian Chanskiï» en 187346. Il nous semble important de souligner que ce nationalisme divergeait du slavophilisme initial, dans la mesure où il s'est toujours maintenu dans un cadre étroitement légitimiste, évacuant les critiques fondamentales de Khomiakov ou des frères Aksakov à l'égard du régime autocratique «inventé » par Pierre-le-Grand, accusé d'être le premier responsable du maintien du servage en Russie.

Figure-clef de cette géographie nationaliste au service de l'Etat, Piotr Pétrovitch Sémionov Tian Chanskiï (1827-1914) ${ }^{47}$ a régné sur les destinées de la Société impériale russe de géographie

\footnotetext{
42 P. P. Sémionov, Istoriza polouviékovoï diéatitiel'nosti Impiératorskovo Rousskovo Guiéografitcheskovo Obchtchestva (Historique de cinquante années d'activité de la Société impériale russe de géographie), St Petersbourg, 1896, 3 t., 1377 p.

${ }^{43}$ L. S. Berg, V siésoïonznoiee Guiéografitcheskoïé obchtchestvo za sto liét (Les cent ans d'existence de la Société de géographie de l'Union soviétique), Moscou-Léningrad, 1946, 263 p.

${ }^{44}$ M. Bassin, «Russian Geographers and the 'National Mission' in the Far East» in D. Hooson, Ex-soviet identities and the return of geography, 1994, p. 112-133.

45 À sa fondation, la quasi-totalité des membres russes de l'IRGO étaient nobles et/ou militaires. Emanation de l'aristocratie, l'institution s'est démocratisée par la suite, suivant vraisemblablement en cela le mouvement général de l'université russe.

46 Wladimir Berelowitch montre que, dans le cas de la section d'ethnographie de l'IRGO, le remplacement des « allemands » a eu lieu plus tôt, dès la fin des années 1840. Les thèses slavophiles sur la narodnost' (nationalité) russe ont alors irrigué les recherches de la section, par l'entremise de Nadiéjdine notamment. Il y a eu recentrage de l'ethnographie qui, délaissant les « groupes ethniques sibériens », s'est focalisée sur l'étude de la terre et du peuple russe.

47 Pour un aperçu plus complet sur la vie et les travaux de Sémionov, voir l'article que lui a consacré Colin Thomas dans Geographers: Biobibliographical Studies, volume 12 (1988), p. 149-158. C'est de loin la meilleure monographie consacrée à un géographe russe dans cette revue. On pourra également se référer aux travaux de W. B. Lincoln : Pëtr Petrovich Semënov-Tian
} 
pendant plus de quarante ans (1873-1914). Issu de la petite noblesse de service «créée » par Pierre-le-Grand, serviteur de l'État impérial par nationalisme et par conviction " pragmatique ${ }^{48}$, il incarne pour beaucoup d'historiographes la paternité (ou la «grand-paternité »!49) de la géographie russe. Sémionov a terminé ses études de géographie et de géologie à Berlin en 18531854. À cette occasion, il a fréquenté K. Ritter et A. von Humboldt. Nombre d'historiographes (à commencer par D. N. Anoutchine ${ }^{50}$ ) ont évoqué ces contacts et les ont commués en une filiation. À notre connaissance, nul n'a véritablement réfléchi à la question, qui mériterait plus que des affirmations rapides. Botaniste et géologue de formation, Sémionov a quelque peu délaissé les sciences naturelles après $1860^{51}$ pour se consacrer, au sein d'institutions officielles, à l'étude de la population russe. En 1858, il avait été coopté à la commission impériale chargée d'étudier les modalités de l'émancipation des serfs ${ }^{52}$, au sein de laquelle il a joué un rôle déterminant. En 1863, il a été nommé à la tête du Comité central de statistique, qu'il allait diriger jusqu'en 1897. Pendant plus de trente ans, il a essayé de fédérer et de rationaliser les publications disparates des ministères. À la fin des années 1850 avaient commencé à être publiées les spravotchno-statistitcheskiiue 'pamiatnyïé knijki', brochures statistiques décrivant un gouvernement (goubiernï̈a) ou une région $(\text { oblast })^{53}$, rédigés par des collaborateurs du gouvernement ou des fonctionnaires locaux. Piotr Sémionov a refondu le genre et lui a donné une forme canonique, l'Annuaire statistique de gouvernement (ou de district). Chaque volume était constitué d'un court texte introductif suivi de données réparties en six chapitres ${ }^{54}$. La matière de ces annuaires a abondamment nourri les ouvrages ultérieurs des Sémionov père et fils, notamment La Russie pittoresque et La Russie. Description complète de notre patrie, ainsi que leurs tentatives de régionalisation de la Russie centrale.

La question de la postérité de Sémionov ne nous semble pas réglée actuellement : n'ayant jamais enseigné, il n'a pas pu former autour de lui une «école » au sens littéral du mot. Demeure son influence capitale au sein de l'IRGO. De 1860, date de sa nomination à la tête de la section de géographie physique, jusqu'à sa mort en 1914, Piotr Sémionov a supervisé l'ensemble des missions scientifiques et exploratoires de l'institution. Nombreux sont les historiographes à avoir insisté sur son rôle de mentor auprès des explorateurs. Il a notamment élaboré des "grilles » visant à systématiser le devisement des régions explorées afin d'obtenir des travaux relativement homogènes par leur contenu. L'intense activité de la Société de géographie lui doit beaucoup : l'IRGO était non seulement le commanditaire, mais aussi l'éditeur des explorateurs du territoire russe. Entre 1846 et 1914, elle a publié pas moins de 10 périodiques, dont les plus durables ont été les Otchtchioty IRGO (Compte rendus de l'IRGO, 1845-1911 et 1915) et les bulletins des trois sections principales (Zapiski IRGO... po obchtcheï guiéografii, po otdiéliéniüon etnografii, po otdiéliéniïion statistiki). Elle a également édité des ouvrages, dont le monumental Dictionnaire géographique et statistique de l'Empire Russe ${ }^{55}$ dirigé par P. P. Sémionov, et patronné de nombreuses traductions,

Shanskii : The life of a Russian Geographer, Nextonville, Mass., 1980 et In the Vanguard of Reform (Russia's enlightened Bureaucrats 1825 1861), DeKalb, Northern Illinois U. P., 1982.

48 Sémionov était en effet convaincu que seul un pouvoir fort pouvait permettre à la paysannerie russe de se libérer de ses entraves serviles. Il nous semble vraisemblable d'affirmer que P. P. Sémionov a été très profondément influencé par les thèses slavophiles, dont on retrouve les principales inflexions chez lui. Il nous semble appartenir à la nébuleuse de ces hommes d'État éclairés qui ont essayé d'infléchir la politique autocratique dans une perspective plus sociale (parce que fascinés par la question de la condition paysanne russe).

49 Ainsi le désigne I. G. Saouchkine dans son Histoire et méthodologie de la science géographique, op. cit.

50 D.N. Anoutchine, «La géographie en Russie » dans A. Soupan, Fondement de la géographie, M.-St P., 1914.

51 Bien qu'il ait animé puis dirigé la section de géographie physique de l'IRGO de 1855 à 1872.

52 Émancipation qui a eu lieu en 1861, avec la promulgation du Statut des paysans libérés du servage.

53 «En général, ces brochures comportaient trois parties. La première donnait quelques informations sur les fondations et l'organisation de la Province. La deuxième était consacrée à des données statistiques disposées sous forme de tables et relatives à la population et à l'économie de la province, parfois assorties d'un commentaire. Dans la troisième étaient publiés différents articles sur l'histoire du gouvernement, sur ses villes et ses hauts-lieux, sur l'économie et l'ethnographie, assortis d'un index bibliographique, ... » dans L. S. Abramov (1972).

54 Conditions naturelles, Population et genre de vie, Agriculture et utilisation du sol, Moyens de production, Organisation économique et budget, Marché et économie de commerce.

55 Guiéografo-statistitcheskï̈ slovar' Rossiïskoï Impiérii, Saint-Petersbourg, 1863-1885, 5 vol. 
essentiellement de l'allemand et du français ${ }^{56}$. Plus surprenante, la traduction en langues étrangères (et notamment en français) d'ouvrages russes, mentionnée par T. Matviéiéva dans son article «Vers une histoire des liens scientifiques franco-russes dans le domaine de la géographie (seconde moitié du XIX siècle - début du XXe siècle) $»^{57}$. Cet important développement éditorial a été amplifié par la multiplication des antennes régionales de l'IRGO ${ }^{58}$, qui à leur tour se sont mises à publier des bulletins (zapiski) et « nouvelles » (izviestia).

Par ses très nombreuses activités, P. P. Sémionov nous apparaît un peu comme l'organisateur et le rationalisateur d'une discipline encore meuble, que son idéologie pétrovienne ${ }^{59}$ a maintenue au service de l'état impérial. À l'aune d'une épistémologie «kuhnienne ", il serait intéressant de s'interroger sur la place de cet administrateur exemplaire dans la cyclique des paradigmes scientifiques. Peut-être nous apparaitrait-il alors comme le pilier d'une "période normale» de la géographie russe, acteur d'une systématisation de ce qui avait été ébauché auparavant par Lomonossov et les Académiciens. À la suite de ces derniers, il aurait rationalisé l'inventaire du territoire impérial et édifié, à partir des années 1870-1880, un premier découpage régional de la Russie. Il nous apparait surtout comme un organisateur institutionnel, fédérant des initiatives éparses sous l'égide de la Société impériale de géographie. Homme d'état, dirigeant de nombreuses institutions semi-officielles, P. P. Sémionov symbolise une géographie enserrée dans la demande étatique, qui ne s'est pas encore autonomisée en «basculant» du côté de la société civile. Pourtant, la sécularisation de la discipline semble s'être amorcée de son vivant et il serait erroné de restreindre l'activité de Sémionov du fait de sa position dans l'appareil d'État. Ses volumineux ouvrages de vulgarisation (le Dictionnaire géographique et La Russie pittoresque avant tout) sont là pour témoigner de sa volonté de voir diffuser la culture géographique au-delà des cercles étatiques qui lui étaient acquis. Plus largement, en l'absence d'un travail définitif sur la question, il est difficile d'évaluer quelle part l'IRGO a prise dans la sécularisation de l'intérêt pour la géographie, sachant ses origines et son recrutement initial.

\section{Géographie et société civile}

En Europe occidentale, l'essor de la discipline universitaire " géographie » dans la seconde moitié du XIX siècle procède d'un intérêt général de la société pour la "chose géographique », support électif de l'aventure coloniale ${ }^{60}$ et du nationalisme. Dans un monde qui s'ouvre et se décloisonne, livré à l'appétit des nations industrialisées, le discours géographique, méthodologiquement pragmatique et fortement descriptif, s'impose comme l'un des outils privilégiés de l'appropriation intellectuelle des régions lointaines. La multiplication des sociétés de géographie, dans les capitales puis en province, et les succès éditoriaux du genre géographique

\footnotetext{
56 Parmi les auteurs traduits, on notera : Ritter (par Sémionov), Richthofen, Supan, Reclus (La Terre et les Hommes et certains volumes de sa Géographie universelle).

57 T. P. Matviéiéva, «K Istorii roussko-frantsouzskikh naoutchnykh sviazieï v oblasti guiéografii (vtoraïa polovina XIX viéka natchalo Xx viéka)» dans Isviestia V.G.O. (Sté. de Géo. de l'U.R.S.S.), 1977, t. 109, p. 525-530. D'après Matviéiéva, il s'agissait essentiellement de compte rendus d'expéditions, qui auraient rencontré un certain succès en France. L'auteur évoque notamment la lettre d'un édile de Paris à l'IRGO, réclamant des publications pour sa bibliothèque de quartier.

${ }^{58}$ Les principales fondations l'ont été à Tbilissi (1851), Irkoutsk (1851), Orenbourg (1867), Omsk (1877) et Khabarovsk (1893). Nous avons recensé un total de quatorze «sections » (otdiély) régionales, mais il y en a peut être eu d'autres. Il faut constater qu'à l'exception de la maison-mère, ces créations se sont faites dans des régions-frontières, à partir de villes-relais de la colonisation russe. Leur implication dans le dispositif colonial ne fait guère de doute...

${ }^{59}$ Idéologie héritée de Pierre-le-Grand, qui finalise l'activité humaine au service de l'Empire russe.

${ }^{60}$ Pour l'Angleterre, of. A. Godlewska \& N. Smith (eds), Geography and empire, Blackwell, The Institute of British Geografers Special Publications Series, Oxford (U.K.), Cambridge (U.S.A.), 1994, 404 p.

Pour la France, cf. V. Berdoulay, La formation de l'école française de géographie, Bibliothèque Nationale, Paris, 1981 ; M.-C. Robic, Du milieu à l'environnement, Pratiques et représentations du rapport homme/nature depuis la Renaissance, Paris, Eds Economica, 1992, p. 133-149; D. Lejeune, Les sociétés de géographie en France et l'expansion coloniale au XIXe siècle, Albin Michel, Paris, 1993, 231 p. et M. Bruneau \& D. Dory, Géographies des colonisations. XVe-XXe siècles, L'Harmattan, Paris, 1994, 420 p.
} 
(récits de voyage, géographies plus ou moins universelles et autres ouvrages de vulgarisation ${ }^{61}$ ) ont amené certains historiographes à parler d'une mode de la géographie, qui s'estomperait au début du siècle suivant. L'important, ici, est qu'il s'agit d'une appropriation de la discipline par la société civile : au Royaume-Uni comme en France, cette vogue a touché les milieux cultivés, indépendamment du projet impérialiste mené par les états. S'agissant de la Russie tsariste, le problème se complique. En effet, la question de l'émergence d'une société civile russe au XIX ${ }^{e}$ siècle est fort épineuse, car difficile à dater et à délimiter. L'opinion publique, concept flou qui recouvre les diverses médiatisations de la société, est ici introuvable, occultée par l'avènement de l'Intelligentsia ${ }^{62}$, incarnation russe de la société civile, même si elle n'en recouvre pas toutes les facettes. Dans les années 1830/40, quelques cercles d'étudiants, étroitement surveillés et régulièrement réprimés, ont été à l'amorce d'une dynamique sociale qui allait s'amplifier et se cristalliser sous le règne d'Alexandre II (1855-1881), puis au tournant du siècle. Une société civile réduite à l'aristocratie et aux citadins instruits a émergé en l'espace d'un demi-siècle, malgré l'hostilité du régime autocratique et le maintien d'une répression politique sourcilleuse. Sujet pensant, cette Intelligensia s'est constituée en relation étroite avec cet autre, objet de toutes ses préoccupations : l'énorme paysannerie russe, référent, modèle, matrice, repoussoir dans de rares cas. Car l'un des aspects fondamentaux de cette opinion russe, outre l'engagement politique, est sa passion pour les questions sociales. Tous les champs du savoir et de l'expression sont finalisés, à la suite de Biélinskiï ${ }^{63}$, au service du social.

Contemporaine de cette éclosion, la Société impériale de géographie a vraisemblablement épousé les débats de son temps. La section d'ethnographie, dont Wladimir Berelowitch a montré le recentrage, dès les années 1850, sur "la terre russe et le peuple russe » (Berg) ${ }^{64}$, a fait de la paysannerie son objet principal. La présence au sein de l'IRGO d'amis et d'héritiers du mouvement décembriste ${ }^{65}$ (Alexandre Mouraviov, Konstantin Arséniev, Nikolaï Nadiejdine) est très éclairante : elle signe la tonalité réformiste et agrarienne de la Société à cette époque. Une étude plus systématique de la diffusion des thèses slavophiles au sein de l'IRGO serait sans doute très féconde. Toutefois, dans la sphère de la géographie stricto sensu, il en a été différemment : les trois axes principaux de la Société, statistique, explorations et géographie physique, l'ont en quelque sorte démarqué des débats de l'Intelligentsia. Institution officielle, alliance de savants et de fonctionnaires, l'IRGO semble s'être volontairement écartée des polémiques les plus vives de son temps, même si, à titre individuel, ses membres les plus prestigieux ont pu donner de la voix ${ }^{66}$. En retour, les grands publicistes ne se sont pas montrés très tendres. À suivre les historiographes soviétiques des années 1970, chacun y aurait été de sa critique acerbe. Biélinskiï aurait écrit un article sur «la mauvaise géographie pour enfants», dans lequel il aurait appelé de ses voeux une géographie qui ne soit pas seulement un agrégat de connaissances hétérogènes, encombré de chiffres et de nomenclatures. Le premier, Herzen aurait fustigé les "géographes réactionnaires», dénonçant en eux l'idée du déterminisme naturel sur les sociétés humaines. Plus nettement,

\footnotetext{
${ }^{61}$ Dans un autre domaine, le succès des romans « itinérants » de Jules Verne (Voyage au centre de la terre, Le tour du monde en 80 jours, Michel Strogoff, etc.) procède d'un phénomène similaire : la fiction produit un idéal-type du désir géographique du siècle, sublimé et épuré.

62 Sur ce concept faussement évident existe une très volumineuse bibliographie. On trouvera des formulations synthétiques dans M. Raeff, Comprendre l'ancien régime russe. État et société en Russie impériale, Paris, 1982. Dans Etre russe au XIXe siècle, Paris, A. Colin, 1974, Alain Besançon s'essaie à une généalogie de l'Intelligentsia très brillante, mais un peu désamorcée par son obsession antirévolutionnaire.

${ }^{63}$ Vissarion Biélinskii (1811-1848), figure majeure de l'hégelianisme russe et critique littéraire remarquable. Interlocuteur de Gogol', découvreur de Dostoïevskiii, il a été, un siècle avant Sartre, le premier théoricien de l'engagement social de l'écrivain. Cf. Isaiah Berlin, Les penseurs russes, Albin Michel, 1984.

${ }^{64}$ Cité par Wladimir Berelowitch dans «Aux origines de l'ethnographie russe : la société de géographie dans les années 18401850 », op. cit., p. 267.

${ }^{65}$ En 1825 avait eu lieu la première rébellion d'envergure contre le régime tsariste, organisée par de jeunes aristocrates, favorables à une monarchie constitutionnelle ou démocrates. L'insurrection du 15 décembre 1825 (d'où le terme de "décembrisme »), férocement réprimée, a eu une postérité symbolique considérable, puisque les générations ultérieures (à commencer par celle de Herzen) en ont fait le point de départ de la tradition révolutionnaire russe, véritable mythe fondateur de celle-ci. ${ }^{66}$ Cf. ce que nous avons dit précédemment des activités politiques de P. P. Sémionov.
} 
Nikolaï Tchernychevskiii67 (1828-1889), dans le sillage de Rousseau et Pestalozzi, se serait beaucoup intéressé à la géographie, en tant que «discipline d'éveil ». Lui aussi aurait critiqué le nomenclaturisme de la géographie et appelé de ses vœux une discipline recentrée sur l'homme et débarrassée de ses influences métaphysiques (i.e. le déterminisme). Cette contribution critique pose problème: compte tenu du statut accordé par l'Union soviétique à ces précurseurs du socialisme russe, il faudrait pouvoir distinguer leur intérêt effectif pour la géographie de ce qui relève de la mythification disciplinaire. Pour cela, un nécessaire retour aux sources s'imposera.

Plus tangible a été la contribution de géographes-révolutionnaires, mineurs en l'un ou l'autre domaine, mais majeurs dans la liaison qu'ils ont réalisée, comme Konstantin Arséniev (1789-1865), Nikolaï Ogarev (1813-1877), Liov Mentchikov (1838-1888) et Piotr Kropotkine (1842-1921). Ami des Décembristes, de Pouchkine et de Joukovskii,, Arseniev a été le premier à critiquer la méthodologie des officiers-géographes. Il a, le premier, tenté un découpage de la Russie en régions économiques, découpage fondé sur une évaluation des ressources naturelles, avec pour horizon leur exploitation rationnelle. Ses travaux initient un retournement de la problématique du déterminisme, en ce sens qu'ils érigent l'homme en agent modificateur des conditions naturelles. On retrouve les mêmes préoccupations chez Nikolaï Ogarev, collaborateur fidèle d'Herzen, qui propose dans son Essai de répartition statistique de l'Empire russe (1847) un schéma de régionalisation rationnelle, à la frontière de l'économie politique et de la géographie. Enfin, L. Mentchikov et P. Kropotkine ont été les collaborateurs d'Elisée Reclus, dont ils partageaient les thèses sur la place de l'homme dans la nature. Il serait tentant de considérer ces figures-charnières dans la perspective d'une bi-polarisation de la géographie russe. En réaction à une géographie " officielle » naturaliste, déterministe, "fataliste ", évidemment pro-impériale et incarnée par l'IRGO, aurait émergé une autre géographie, inspirée par les grands penseurs contestataires, résolument anti-déterministe et recentrée sur la géographie des hommes. Cette deuxième voie, tracée dans le sillage de Georges P. Marsh, K. Arséniev et Elisée Reclus, trouverait sa figure ultime en Alexandre Ivanovitch Voïeilkov (1842-1916). Climatologue de formation, voyageur impénitent, polyglotte exceptionnel, ce dernier est venu à la géographie dans les années 1880 par l'étude de la désertification en Asie Centrale et dans le Caucase. Voïeïkov a été l'un des premiers à s'intéresser aux effets entropiques de l'action humaine, écologiste (dans l'acception actuelle du terme) avant l'heure. En 1904, il a publié une étude sur «le rôle mondial de l'Océan Pacifique, [puis] un article très remarqué (...) sur les conditions de la rénovation de la Russie » ${ }^{68}$. On lit ici le repositionnement du climatologue, qui devient "géographe complet ${ }^{69}$ dans une perspective résolument humaniste et réformatrice (sous le double signe de l'écologisme et du réformisme social). Homme remarquablement doué et quelque peu visionnaire, Voïeikov est malheureusement demeuré en marge de la géographie de son temps. Il n'a jamais bénéficié d'une quelconque position institutionnelle au sein de la discipline, il n'a pas fait école et son écologisme avant-gardiste, inconciliable avec la vision prométhéenne des staliniens, lui a valu un très long purgatoire comme géographe. À l'étranger, ses nombreuses publications en français et en allemand n'ont pas suffi à lui procurer une stature de géographe majeur et il est quelque peu tombé dans l'oubli. Espérons que cette injustice sera réparée dans un futur proche et qu'une étude d'envergure nous permettra de mieux connaitre ce penseur très original. Voïeïkov ou le paradigme perdu de la géographie russe.

\footnotetext{
${ }^{67}$ Célèbre publiciste russe. Il a joué un rôle déterminant dans la radicalisation de l'Intelligentsia. Contemporain de Nétchaïev, il fut l'un des inspirateurs des groupes de terroristes actifs sous le règne d'Alexandre II, notamment Ziemlia i volia. Dans son roman Le Don, V. Nabokov a écrit une fascinante biographie parodique de Tchernychevskiï, qui restitue le climat de sérieux exalté (et totalement dénué d'humour) de sa génération, celle des «fils» de Tourguéniev. Cf. également Isaiah Berlin, op. cit.

${ }^{68}$ Extrait de "Nécrologie: A. Woeikof », Annales de Géographie, 1916, p. 150-151. Il est important de noter qu'en Russie, c'est Véniamine Sémionov, fils de Piotr, qui rédigea la notice nécrologique parue dans le numéro du 30 janvier/12 février 1916 du journal Novoüé Vriémia (Les Temps Nouveaux).

${ }^{69}$ «Nécrologie... », op. cit., p. 150.
} 
La diffusion de thématiques géographiques dans l'Intelligentsia est difficilement compréhensible si l'on ignore tout de l'organisation des cercles intellectuels russes de l'époque. En l'absence d'une diffusion sans entraves des idées et écrits, une bonne partie des échanges intellectuels devait s'effectuer par des biais privés : réunions d'amis, fêtes, banquets, où se retrouvaient les membres de cercles d'affinités, plus ou moins affichées selon leur degré de politisation ${ }^{70}$. En dépit de la relative libéralisation du régime entre 1855 et 1870, ce fonctionnement s'est maintenu durant le règne d'Alexandre II et s'est même étendu et démocratisé. Le cloisonnement relatif inhérent à cette situation explique, dans la sphère géographique, que se soient développés deux hauts-lieux de la discipline quelque peu concurrents, ou du moins très distincts: Saint-Petersbourg et Moscou. À l'écart du monde d'officiers et de hauts fonctionnaires de la ville impériale, les cercles universitaires moscovites ont cultivé leur différence. Déjà, en 1844-1847, l’université de la vieille métropole avait confié des cours de géographie à A. P. Efrémov, finalement congédié par les autorités impériales en raison de ses amitiés slavophiles. L'impulsion majeure est venue de Nikolaï Frolov, ancien élève de Ritter à Berlin et ami de Granovskiii ${ }^{71}$, initiateur d'un cercle de géographie qui durant les années 1850 publia une revue, le Magazine de géographie et des explorations. Fait décisif, ce groupe moscovite s'est maintenu en dehors du giron de l'IRGO, manifestant une autonomie dont les soubassements géographiques, politiques et sociaux mériteraient plus ample examen. Et c'est à Moscou, trente ans plus tard, que s'est ouverte la première chaire de géographie, détenue par Dimitri Anoutchine, dont on devine les sympathies réformatrices, peut-être inspirées d'Herzen.

L'idée d'une double polarisation, idéologique et géographique, de la géographie russe est séduisante ; elle n'en demeure pas moins sujette à caution. Il n'y a jamais eu de frontière étanche entre les uns et les autres, comme en témoignent les collaborations à l'IRGO d'Anoutchine d'une part, de Kropotkine et Voïeïkov d'autre part. Il faut rappeler également que K. Arséniev avait participé à la fondation de la Société en 1845. La circulation des idées à l'intérieur et à l'extérieur des cercles de savants et de publicistes concernés par la géographie semble s'être effectuée sans créer de confrontations radicales. Demeure l'idée d'un intérêt général de l'Intelligentsia pour des thématiques géographiques, qui à l'époque n'étaient peut-être pas toujours estampillées du label "géographie ». Certains historiographes évoquent une popularité de la discipline dans l'ensemble de l'opinion publique dans les dernières décennies du siècle, mais la mesure historique de ce phénomène n'a encore jamais été réalisée. Les grands ouvrages de vulgarisation des Sémionov père et fils, La Russie pittoresque et La Russie. Description complète de notre patrie sont peut-être le fruit de cet engouement de l'opinion. Des explorateurs comme Prjéval'skiï ou Vladimir Arséniev ont acquis une notoriété extraordinaire et leurs livres ont été des succès de librairie. En somme, en Russie comme ailleurs, la dimension aventureuse des explorations géographiques « coloniales » a contribué à l'audience de la discipline. Soulignant le caractère probable de cet engouement pour la géographie, nous avons aussi le souci d'indiquer qu'il s'agit d'un front pionnier de l'historiographie, jusqu'ici négligé par les spécialistes de la question, qu'ils soient russes ou étrangers.

En Russie comme en Angleterre ou en France, le développement universitaire de la géographie a eu lieu bien après l'élévation de son audience sociale, le discours savant venant en quelque sorte ratifier et normaliser des productions cognitives spontanées de la société. L'institutionnalisation que l'on constate a-t-elle débouché ici aussi sur l'élaboration d'un

\footnotetext{
${ }^{70}$ Dans les mémoires d'Alexandre Herzen, Passé et méditations, L'Age d'Homme, t. 1 \& 2, on trouve une évocation très précise du fonctionnement de ces cercles.

71 Timofei Nikolaiévitch Granovskii (1813-1855) fut dans sa jeunesse l'une des figures majeures de l'occidentalisme et l'ami de Herzen et Ogarev. Professeur d'histoire à l'université de Moscou, il jouissait d'une réputation extraordinaire. Plus libéral et idéaliste que socialiste, il rompit avec Herzen lors de la parution des écrits d'immigration de ce dernier. Il demeure comme l'une des grandes figures intellectuelles du libéralisme russe.
} 
paradigme fort? Peut-on dire qu'à la veille de la Révolution d'octobre, la géographie russe avait acquis une assise et une légitimité équivalentes à celles de ses consoeurs occidentales ?

\section{Une institutionnalisation ébauchée}

La première véritable chaire universitaire de géographie a été créée à Moscou fin 1884 pour Dimitri Nikolaiévitch Anoutchine (1843-1923). Eu égard à la trajectoire scientifique de ce dernier, ce choix pourrait paraittre surprenant.

Anoutchine a commencé ses études universitaires à la faculté d'histoire et de philologie de Saint-Petersbourg. D'après Berg, il a suivi des cours dans des domaines remarquablement divers (de la littérature au droit criminel, en passant par l'histoire et la zoologie). Du printemps 1861 à l'été 1863, il a effectué un long séjour à l'étranger, essentiellement à Rome. Son retour en Russie a été l'occasion d'un revirement décisif: il a abandonné la faculté d'histoire et de philologie de Saint-Petersbourg, déménagé à Moscou et entamé des études de zoologie, qu'il a achevées en 1867. Par la suite, Anoutchine a publié quelques articles de zoologie, avant d'amorcer un nouveau virage en 1874. Cette année-là, il a publié un article très remarqué sur «Les singes anthropomorphes et leur rôle dans la naissance de l'humanité », qui consacre l'influence capitale de Darwin sur toute sa carrière. Délaissant la zoologie, Anoutchine s'est alors tourné vers l'anthropologie et l'ethnologie. En 1875, il a été élu secrétaire de la section d'ethnologie de la Société impériale des amateurs de sciences naturelles, d'anthropologie et d'ethnologie (Obchtchestvo Lioubitiélieï Estiestvoznaniz̈a, Antropologuïa $i$ Etnografïa, abrégé OLEAE), dont il était un membre actif depuis son retour d'Italie. Ce repositionnement disciplinaire lui donnait l'occasion d'occuper la chaire d'anthropologie que l'université de Moscou comptait créer. À cette fin, il est reparti en Europe occidentale (Angleterre, Belgique, France) afin de compléter sa formation. À Paris, il a fréquenté Chantre, Garrigou et l'école d'anthropologie du Museum d'histoire naturelle. Revenu à Moscou, il a été chargé du premier cours d'anthropologie professé en Russie (janvier 1880), avant de soutenir sa thèse magistrale: Sur quelques anomalies du crâne humain (1881). À la même époque (1879-1884), Anoutchine avait commencé à enseigner les sciences naturelles à l'Institution de jeunes filles Sainte-Catherine de Moscou et était devenu un lecteur assidu de Reclus. En 1882, la section d'ethnographie de l'IRGO l'envoya en mission dans le Daghestan pour étudier la langue et les coutumes de l'aoul de Koubatch. En novembre 1884, l'université de Moscou créait la première chaire de géographie et d'ethnologie russe, occupée par... Dimitri Nikolaiévitch Anoutchine. Entre 1885 et 1887, l'intéressé a donc déployé son énergie à enseigner la géographie, avec une nette prédilection pour la géographie physique et la méthodologie ${ }^{72}$. Son premier cours consacré à la géographie de la Russie date de 1887 et, en 1888, il a professé le premier enseignement universitaire russe d'ethnographie. Le point d'aboutissement de cette carrière sinueuse a été l'élévation d'Anoutchine au titre de docteur en géographie honoris causa en 1889 (il avait alors 46 ans). Un an auparavant, la chaire qu'il occupait à la faculté de philologie avait été transférée à la faculté des sciences, décision hautement symbolique mais encore mystérieuse quant à son origine ${ }^{73}$. Pendant trente-et-un ans, il a été le seul et unique détenteur d'une chaire de géographie en Russie. Il a été le passage obligé de plusieurs générations d'étudiants en sciences naturelles, dont il a pu infléchir la trajectoire. En 1894, il a été à l'origine de la création de la revue Ziemliéviédiénizé (Géographie générale), émanation de l'OLEAE qui allait vite devenir une revue de référence (en géographie physique surtout). Il en a été le rédacteur en chef jusqu'à sa mort, en 1923.

La trajectoire universitaire de Dimitri Anoutchine est assez fascinante dans ses revirements et ses repositionnements successifs. L'historiographie soviétique n'ayant rien à nous apprendre

\footnotetext{
72 Son premier cours, fondé sur des travaux de Ritter, Supan et Richthofen, s'est intitulé « Quelques problèmes de la géographie ».

73 Aucun des historiographes soviétiques consultés n'explique les raisons de ce transfert et nous ignorons quelle a été la position d'Anoutchine à cette occasion.
} 
qui puisse éclairer ce chemin, nous en sommes pour l'instant réduits à quelques conjectures qui mériteraient un plus ample examen. Il semble difficile de trouver une cohérence épistémologique à tous ces rebondissements. Aussi, l'hypothèse de choix carriéristes (sans nuance péjorative) nous semble la plus vraisemblable. Doté de connaissances encyclopédiques, nourri par l'évolutionnisme ambiant, Anoutchine s'est retrouvé à la croisée des chemins alors que trois disciplines, l'anthropologie, l'ethnologie et la géographie, amorçaient leur institutionnalisation au sein de l'université russe la plus résolument «moderniste ». Le même homme les a incarnées successivement, choisissant finalement celle qui semblait la plus susceptible de lui assurer une audience (ou une carrière ?) dans le contexte de l'époque, et sans totalement abandonner les deux autres $^{74}$. Il faut aussi souligner que ces disciplines étaient toutes les trois situées à l'interface nature/société, et procédaient fortement du paradigme darwinien qui dominait alors l'université moscovite.

Lecteur assidu des grands géographes allemands de la seconde moitié du XIX siècle (Richthofen, Wagner, Hettner, Ratzel), Anoutchine a épousé la plupart de leurs thèses ${ }^{75}$. Mais sa position quant aux limites de la discipline n'a pas la netteté que l'on trouve chez ses contemporains F. Ratzel ou P. Vidal de la Blache. Là où ces derniers tranchent et s'affirment, Anoutchine préfère citer, glissant d'un auteur à un autre, hésitant entre une conception très large de la géographie, «complexe (...) de sept sciences ${ }^{76}$ » qui rend compte de la totalité de la surface terrestre, et une conception plus restreinte, qui correspondrait globalement à celle des vidaliens. Il y a toujours un certain flou et comme un flottement épistémologique chez lui, qui contraste avec l'attitude d'autres grands fondateurs de sa génération. S'agissant du rapport Homme/Nature, on retrouve dans ses écrits la position œcologique (Vidal, 1903) dominante à l'époque, qui considère l'homme comme une partie intégrante de la Nature, et déterminée par elle. Mais son adhésion initiale aux vues de l'anthropogéographie ratzelienne, encore nette en 1893, a progressivement cédé la place à une conception plus dialectique. Ainsi, en 1912, le déterminisme naturel n'est plus chez lui qu'une posture de principe, l'essentiel étant ailleurs, dans l'action modificatrice de l'Homme, qui transforme son milieu, y compris le climat (référence implicite à Voïeïkov ?). Ici encore, il faut noter l'absence de dogmatisme d'Anoutchine, qui aux phrases tranchées du tribun, préfère toujours les citations et l'effacement du rédacteur. Son abondante production épistémologique ${ }^{77}$ nous le révèle tout à la fois lecteur assidu des autres, diffuseur d'idées, pédagogue, mais également défenseur d'une géographie très physicienne ${ }^{78}$. Demeure alors la

\footnotetext{
${ }^{74}$ Cette hypothèse tend à confirmer, pour la Russie, les thèses d'Horacio Capel, développées dans l'article « Institutionalization of geography and strategies of change », p. 37-69, in Stoddart, D.P., (ed.), Geography, Ideology \& Social Concern, 1981, Oxford, Blackwell, VI-250 p. L'auteur souligne le rôle moteur des stratégies de pouvoir et des opportunités professionnelles dans l'institutionnalisation universitaire de la géographie dans la seconde moitié du XIXe siècle. Il insiste sur le caractère hybride et fort divers des curricula des géographes promus à la tête de la discipline universitaire, en somme géographiquement impurs. Dans le cas d'Anoutchine, on retrouve bien ces caractéristiques.

${ }^{75}$ Cf. notamment ses deux longs articles intitulés « Géographie » dans les Encyclopédies Brokhaus et Efron (Saint-Petersbourg, 1892, vol. VIII) et Granata (Saint-Petersbourg, 1912, t. 13). Dans le second, il se réclame également de Brunhes, qui deux ans auparavant avait publié son opus La géographie humaine. Essai de classification positive. Principes et exemples.

76 Anoutchine les nomme Géographie astronomique (ou mathématique), géophysique, géographie physique, géographie biologique, anthropogéographie, géographie régionale et géographie historique, et chacune d'entre elles entretient des rapports complexes avec les sciences voisines de la géographie.

77 Outre les deux articles op. cit., on pourra consulter notamment: "Niéskol'ko slov o razvitii rouskovo ziemliéviédiéniïa i zadatchakh guiéografitcheskovo kroujka v Moskvié » (Quelques mots à propos du développement de la géographie russe et des missions du cercle de géographie de Moscou), Ziemliéviénieé, M., 1894, I, p. 78-126, "O priépodavanii guiéografii i o voprossakh s nim sviaziannym» (À propos de l'enseignement de la géographie en Russie et des questions afférentes), reproduit dans

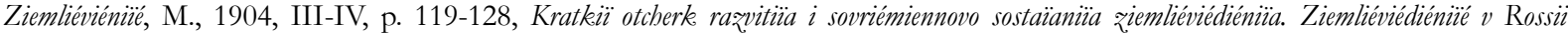
(Bref essai sur le développement et la situation actuelle de la géographie. La géographie en Russie), Moscou, 1914, et bien d'autres encore...

78 Compte tenu du cursus d'Anoutchine, cette position pourrait paraitre curieuse. Ce paradoxe apparent a été remarquablement analysé par Horacio Capel (1981) : "This explain why, surprisingly, some of the new geography professor tended to work on aspects of geography totally different from those of their prior education : ethnographers or historians became physical geographers, geologists developed the human aspects of the new science (...); this would, however, need careful balancing, as it was always necessary to justify the new science and emphasize the difference between it and their old done. » (p. 63).
} 
question de sa contribution personnelle à la pensée et aux thématiques de la géographie de son temps...

Ce qui frappe le plus chez Anoutchine est sa capacité à susciter de nouveaux champs de recherche. Son article intitulé «Le relief superficiel de la plaine européenne de Russie, vu à travers ses représentations successives », bien qu'essentiellement historique dans son contenu, a été le point de départ de nombreuses recherches morphologiques. Il est aussi le fondateur de la limnologie et de la géographie des lacs en Russie. Dans l'ensemble de ces domaines, son mérite essentiel est celui de l'inspirateur : il a émis quelques hypothèses fondatrices, avant de convaincre ses étudiants d'approfondir la question, avec publication dans Ziemliéviédiénizé à la clef. Plus que le fondateur d'un paradigme géographique, Anoutchine nous apparait comme un accoucheur de recherches et un remarquable comparatiste, capable de s'emparer de n'importe quel sujet pour établir force parallèles et associations. Ses travaux en archéo-ethnographie sont à ce titre exemplaires: il y déploie une grande intelligence observatrice, linguistique et comparative ${ }^{79}$. La liste des sujets sur lesquels il a écrit est vertigineuse. Il a rédigé dans Ziemliéviédiéniié un «feuilleton géographique » sur Le Japon et les Japonais ${ }^{80}$, ultérieurement repris en volume. Il s'est fait le chroniqueur de nombreuses expéditions polaires. Il s'est passionné pour l'étude des catastrophes naturelles (tremblements de terre, volcanisme, inondations). Il a multiplié les textes de réflexion sur la géographie et son histoire. Il a publié de nombreux articles monographiques consacrés à des massifs montagneux. Il s'est beaucoup intéressé aux phénomènes de migration ("Les Allemands en Amérique », "Les migrations aux Etats-Unis », etc.). Il est passé maittre dans l'art de la nécrologie (Prjéval'skiï, Reclus, Richthofen, etc.). Enfin, il a écrit une série d'articles sur Pouchkine dans les Rousskiié Viédomosti (le Bulletin Russe), revue dont il fut l'un des fondateurs en 1881. Encore n'avons-nous mentionné que les sujets récurrents...

À la lumière de ces quelques indications biographiques se pose inévitablement la question du rôle d'Anoutchine dans l'évolution scientifique et institutionnelle de la géographie en Russie. Notre hypothèse est que, professeur d'université, pédagogue, vulgarisateur, journaliste ${ }^{81}$, il aurait procuré à la discipline une assise institutionnelle et une voix, à défaut de lui tracer une voie... En effet, à la différence de Ratzel, Vidal ou Mackinder, il n'a pas laissé derrière lui de réflexion théorico-épistémologique originale; dès lors, il est difficile de le considérer comme un chef d'école complet, et ceci malgré sa position monopolistique à la tête de la géographie universitaire russe de 1884 à 1916. Plus qu'un novateur, plus qu'un chercheur, Anoutchine nous apparaitrait comme un lettré, un homme de cabinet, doté d'un remarquable esprit de synthèse et de diffusion. Parallèlement à Véniamine Sémionov, il a contribué à introduire les principales grilles d'analyse de la géographie occidentale en Russie, essentiellement auprès et par l'entremise de ses étudiants. Et c'est de ce vivier qu'ont émergé les premiers grands noms de la géographie soviétique (Berg, Kruber, Krasnov, Glinka, Grigoriev, etc.). Ce sont eux qui ont développé l'école «zonalepaysagère » ou Landchaftoviédiéniié, paradigme distinctif de la discipline dans l'ex-Union Soviétique. Sur le plan strictement scientifique, l'approche de ces chercheurs procède davantage des travaux de Dokoutchaïev sur la zonation des sols, voire du systémisme voïeïkovien, que de la géographie protéiforme d'Anoutchine, leur maitre désigné.

Les modalités d'émergence du paradigme zonal-paysager soviétique sont encore très obscures. En effet, ce qui s'est passé entre 1914 et 1930 dans la géographie russe est un mystère. Le développement des chaires, le mouvement social au sein de la communauté scientifique, la catalyse des idées, en somme tout ce qui donne de l'épaisseur à l'histoire disciplinaire, nous

\footnotetext{
79 Cf. notamment «Sani, lad'ia i koni kak prinadliejnosti pokhoronovo obriada » (Traineaux, barques et chevaux, éléments constitutifs des rites funéraires) et « $\mathrm{K}$ istorii oznakomliéniïa s Sibiriou do Iermaka » (À propos de l'histoire de la connaissance de la Sibérie avant Ermak), deux articles publiés en 1890.

80 Les circonstances ne sont pas neutres : les trois articles qui constituent cet ouvrage ont été publiés en 1904 pour les deux premiers et en 1906 pour le dernier, ils « encadrent » de ce fait la guerre russo-japonaise de 1904-1905.

81 Essentiellement pour le Courrier russe de 1879 à 1881 puis au Bulletin russe, op. cit., jusqu'en 1912. Mais il a écrit de très nombreux articles pour d'autres journaux.
} 
demeure quasiment inconnu. S'agissant d'Anoutchine lui-même, l'essentiel est voilé. Nous ne faisons qu'entrevoir ses engagements pré-révolutionnaires (réformistes ?). Nous savons qu'il s'est rallié au nouveau régime (mais jusqu'où est allé son ralliement ?). Nous savons qu'il a continué à occuper des places honorifiques (notamment à la tête de l'OLEAE) jusqu'à sa mort, en juin 1923. Nous voyons ses disciples occuper très rapidement les places maittresses des universités et des instituts de géographie créés par le nouveau pouvoir. Nous assistons enfin à la rapide canonisation du Maitre peu après sa mort. Tout ceci est fort intrigant et met en évidence un enjeu historiographique majeur, celui de la soviétisation de la géographie russe et des reclassements institutionnels et sociaux qui l'ont peut-être émaillée : enjeu majeur, mais pour l'heure encore inexploré.

En définitive, à la veille de la Révolution de 1917, la géographie n'avait pas encore vraiment diffusé dans l'enseignement supérieur, la communauté scientifique n'avait pas définitivement cristallisé et les pratiques de recherche n'avaient pas été normalisées par la définition d'un paradigme de référence. L'institutionnalisation de la géographie n'était pas achevée et ne représentait encore qu'une ébauche, que la période soviétique refaçonnerait. 
Née sous les auspices de l'expansionnisme pétrovien et de l'esprit des Lumières (Lomonossov), la géographie russe a été marquée durant toute son histoire pré-révolutionnaire par un très fort pragmatisme de projet. Plus qu'une science, elle a longtemps fourni un cadre, un nom, une justification au projet d'expansion territoriale grand-russe. À ce titre, elle a été largement incarnée par des militaires, des fonctionnaires et des explorateurs, géographes occasionnels et figures populaires d'une odyssée nationale et nationaliste (Nikolaï Prjéval'skiï, Piotr Sémionov, Vladimir Arséniev et tant d'autres...). Il a fallu attendre 1845 et la création de la Société impériale russe de géographie pour que s'amorce la disciplinarisation de pratiques jusque là éparses, véritable acte de naissance officielle. À cette occasion, le pouvoir étatique réaffirmait son besoin informatif et l'adéquation du vocable géographique à cette demande, consacrant la souplesse et le caractère œcuménique d'un champ intellectuel capable de fédérer sous son nom explorations, géodésie, cartographie, recensements, statistique, sciences naturelles et ethnographie. Cette diversité d'approches a trouvé son médium dans les grands formulaires d'enquête de l'IRGO et des administrations des Goubiernii (gouvernements). Elle y a également trouvé ses limites (bureaucratiques ?), dénoncées par l'Intelligentsia et certains géographes. Car c'est aussi en ces années d'affirmation que la géographie a connu un certain succès dans l'opinion, porté, motivé par les récits des explorateurs et le contexte colonial. Il a malgré tout fallu attendre la presque fin du siècle (1884-85) pour que l'université légitime et ratifie un développement jusque là extra-académique. Pour des raisons encore obscures, l'institutionnalisation de la discipline n'a plus connu de nouvelles avancées avant l'installation au pouvoir des Bolchéviks. Ceci n'a pas empêché la géographie russe de progresser, par l'entremise de ses grands chercheurs «transfuges » (Piotr Sémionov dans les années 1850, Voïeïkov et Anoutchine trente ans plus tard et Dokoutchaïev malgré-lui) ou "originaires » (Véniamine Sémionov, Berg, de générations plus tardives, filiales en quelque sorte). Malgré la valeur de ces contributions diverses et la fécondité des recherches géographiques, la discipline ne s'était pas encore dotée, en 1917, d'une voie spécifique aisément désignable comme École russe. La coalescence des approches et des voies s'est effectuée durant l'époque troublée de la première décennie de l'U.R.S.S., et demeure très mal connue. C'est pourtant alors qu'a émergé le paradigme zonal-paysager qui allait dominer la quasitotalité de la géographie (physique) soviétique, propagé par les élèves d'Anoutchine, héritiers de Dokoutchaïev et très bien intégrés dans le dispositif universitaire créé par le régime soviétique.

Les mêmes hommes, fondateurs de la discipline et principales figures institutionnelles, en ont été également les premiers commentateurs (Anoutchine d'abord, puis Berg et Grigoriev), dans une perspective plus ou moins mémorialiste. Puis, à partir des années 50 , la production historiographique a connu un développement remarquable, principalement à destination du public étudiant, auquel il fallait donner une image stable et légitimante de l'histoire disciplinaire. C'est dans ce contexte qu'ont émergé les premiers vrais historiens de la géographie russe, que celle-ci soit pour eux un miroir pour polémiquer (Iouri Souchkine, Vsiévolod Anoutchine) ou un objet d'étude à part entière (Liov Abramov, Tatiana Matviéiéva). En dépit ou en raison de cette volumineuse historiographie, la géographie russe conserve bon nombre d'aspects intrigants et non-explorés. La mise en oeuvre d'analyses socio-historiques n'en est qu'à ses débuts et demande désormais une confrontation directe avec la matière d'origine, textes, sources, archives, documents privés, afin de résoudre, entre autres, certaines des questions formulées dans les pages qui précèdent, parfois aussi pour les invalider ou les reformuler. À ce titre et pour des raisons évidentes, l'énigme majeure réside dans ces années troublées qui s'étendent de 1914 à l'instauration du stalinisme (1928/1930). Quelque chose s'est alors noué, qui a façonné la géographie soviétique sur les plans les plus divers. Ici, l'histoire disciplinaire rencontre l'un des moments capitaux du $\mathrm{XX}^{\mathrm{e}}$ siècle. Et là où un pays entier a été transformé, bouleversé, la communauté géographique n'aurait qu'imperceptiblement bougé, quelques marginalisations mises à part. Que dire de cette étrange quiétude : ralliement généralisé ou miroir trompeur? 